\title{
Corrupción y desarrollo en China y América Latina
}

\section{Corruption and Development in China and Latin-America}

DOI: $10.32870 /$ mycp.v9i27.684

\author{
Héctor Flores Márquez ${ }^{1}$ \\ Ana Lilia Valderrama Santibáñez ${ }^{2}$ \\ Omar Neme Castillo ${ }^{3}$
}

\begin{abstract}
Resumen
No hay un consenso claro sobre los efectos de la corrupción en distintas dimensiones económicas, aunque generalmente se percibe como dañina para variables como crecimiento o IED. El objetivo de este artículo es determinar el efecto de la corrupción en el desarrollo humano en América Latina y China. Se sigue una metodología de panel de datos estática con la transformación de Prais-Winsten con errores estándares corregidos para paneles correlacionados (PCSE), y de datos de panel dinámica del método generalizado de momentos (GMM) y sys-GMM para 18 países latinoamericanos y China para el periodo 2001-2019. Se demuestra que la corrupción funge como limitante del desarrollo para la mayoría de los países de la muestra. Sin embargo, para algunos países como China se encuentra que la corrupción tiene un efecto positivo. Asimismo, se encuentra diferencia significativa en la sensibilidad del desarrollo a la corrupción entre los países.
\end{abstract}

Palabras clave: corrupción, América Latina, China, IDH, IPC.

\begin{abstract}
There is no clear consensus on the effects of corruption in different economic dimensions, although it is normally perceived as harmful for variables such as growth or FDI. The aim of the paper is to determine the effect of corruption on human development in Latin America and China. The methodology employed is a static data panel with the Prais-Winsten transformation with corrected standard errors for correlated panels (PCSE), and dynamic panel data from the Generalized Method of Moments (GMM) and sys-GMM, for 18 Latin American countries and China for the period 2001-2019. It is shown that corruption serves as a development constraint for most of the countries in the sample. However, for some countries like China, it is found that corruption has a positive effect. There is also a significant difference in the sensitivity of development to corruption among countries.
\end{abstract}

Keywords: corruption, Latin-America, China, HDI, CPI.

Artículo recibido el 02 de marzo de 2020 y dictaminado el 18 de mayo de 2020.

1. Estudiante del Doctorado en Ciencias Económicas. Instituto Politécnico Nacional. Plan de Agua Prieta 66, Col. Plutarco Elías Calles, Miguel Hidalgo, Ciudad de México, México. C.P. 11350. ORCID: https://orcid.org/0000-0002-1766-5266 Correo electrónico: hfmarquez@hotmail.com

2. Instituto Politécnico Nacional, Escuela Superior de Economía, Sección de Estudios de Posgrado e Investigación. Plan de Agua Prieta 66, Col. Plutarco Elías Calles, Miguel Hidalgo, Ciudad de México, México. C.P. 11350. ORCID: https://orcid.org/0000-0003-0372-7099 Correo electrónico: avalderrama@ipn.mx

3. Instituto Politécnico Nacional, Escuela Superior de Economía, Sección de Estudios de Posgrado e Investigación. Plan de Agua Prieta 66, Col. Plutarco Elías Calles, Miguel Hidalgo, Ciudad de México, México. C.P. 11350. ORCID: https://orcid.org/0000-0001-8509-7937 Correo electrónico: oneme@ ipn.mx 


\section{Introducción}

La corrupción se presenta de manera cotidiana en las transacciones económicas y de muy distintas formas a través de actos pequeños, medianos y grandes en la interacción entre los distintos niveles de gobierno y el sector privado. La razón central de este fenómeno es que los gobernantes no actúan maximizando el bienestar social, sino que persiguen objetivos propios (Hopkin \& Rodríguez-Pose, 2007), representando una falla del gobierno desde una perspectiva clásica de la economía.

En la literatura del tema existen distintas visiones respecto al impacto de la corrupción en la economía, sin que exista claro consenso. Por un lado, el enfoque "grabbing hand" subraya las consecuencias negativas de la corrupción, derivada principalmente de altos niveles de intervención gubernamental en la economía. En términos de Popova y Podolyakina (2014), esta visión señala el carácter destructivo de la corrupción en indicadores económicos. Por ejemplo, Cieslik y Goczek (2018) sugieren que este fenómeno obstaculiza directamente el crecimiento económico al limitar las inversiones. En particular, Mo (2001) estima que un aumento del $1 \%$ en este tipo de actos reduce la tasa de crecimiento económico en alrededor de $0.72 \%$. Cooray y Dzhumashev (2018) sostienen que reduce indirectamente la oferta de trabajo, aumenta la carga impositiva y la economía informal. Pieroni y d'Agostino (2013) argumentan que tiene efectos en términos de libertad económica a nivel micro. En general, el impacto negativo se estima en distintas variables como el Producto Interno Bruto (PIB) per cápita (Lucic, Radisic \& Dobromirov, 2016); la inflación (Blackburn \& Powell, 2011); la productividad total de los factores (Wu, Li, Nie \& Chen, 2017); la Inversión Extranjera Directa (IED) (Delgado, McCloud \& Kumbhakar, 2014) e indirecta (Jain, Kuvvet \& Pagano, 2017); la deuda pública (Cooray, Dzhumashev \& Schneider, 2017); las ventas reportadas sujetas a impuestos (Alm, Martínez-Vázquez \& McClellan, 2016); y el tipo de cambio (Bahmani-Oskooee \& Nasir, 2002).

Por otro, el enfoque "helping hand" afirma que los efectos positivos de la corrupción sobrepasan a los costos, por lo que tiende a favorecer a la economía y, por tanto, a que el fenómeno se mantenga en el tiempo. Para Dzhumashev (2014), la corrupción mejora la eficiencia económica cuando el tamaño del gobierno está por arriba del nivel óptimo, lo cual implica que existe un nivel de corrupción maximizador del crecimiento. Una idea similar se discute en Saha y Gounder (2013), quienes estiman una relación positiva 
entre corrupción e ingreso. Asimismo, Larsson (2006) indica que la corrupción es compatible con un rápido crecimiento en China. Aún más, para Jiang y Nie (2014) el milagro chino de elevado crecimiento se explica por los altos niveles de corrupción —evasión de regulación gubernamental-. De hecho, la corrupción está en el centro de la "paradoja del este asiático", puesto que las economías con crecimiento excepcional se asocian con culturas corruptas. Rock y Bonnett (2004) y Huang (2016) rechazan la percepción común sobre la corrupción como dañina para el crecimiento económico.

En línea con esto, Trang, Papanastassiou y Nguyen (2017) argumentan que niveles bajos de corrupción en el país anfitrión de capitales extranjeros se asocia positivamente con primas de cobertura en periodos posteriores a la crisis. También, Ahlin y Pang (2008) señalan que niveles bajos de corrupción son positivos para la economía cuando se acompañan de reformas financieras. Para Jiang y Nie (2014) los efectos positivos se sienten a nivel regional puesto que la corrupción impulsa la rentabilidad de las empresas privadas y contribuye a su crecimiento (Wang \& You, 2012). Por último, Quazi, Vemuri y Soliman (2014) indican que en economías en desarrollo, caracterizadas por marcos regulatorios débiles, hay evidencia de una relación positiva entre corrupción-IED, aunque reconoce que en la medida que introduzcan reformas que lo fortalezcan, pueden revertirse dichos efectos.

No obstante, una parte de la literatura es no concluyente, con resultados variados. Más precisamente, Tiberiu, Tamasila y Taucean (2016) exponen que la corrupción influye en la actividad de emprendimiento, pero no ejerce efecto en las conductas evasivas al fisco. Méndez y Sepúlveda (2006) indican que el nivel de corrupción que maximiza el crecimiento es significativamente mayor a cero, con efectos positivos para bajos niveles y con negativos para alta incidencia de corrupción. Considerando el papel de las instituciones políticas, Aidt, Dutta y Sena (2008) sostienen que, en regímenes de alta calidad, la corrupción tiene impacto sustancialmente negativo en el crecimiento, pero en regímenes de baja calidad no hay impacto evidente.

Además, Policardo y Sánchez (2018) afirman que la corrupción no parece tener efecto significativo en la desigualdad de ingresos, pero establecen que la desigualdad tiende a generar este comportamiento social. Para Talvitie (2017), a pesar de la corrupción generalizada y débiles instituciones, las economías asiáticas emergentes lograron atraer IED, crecimiento económico y reducción de la pobreza; mientras que esto no ha sucedido en otras economías en desarrollo. 
En concreto, Bose, Capasso y Murshid (2008) argumentan que la corrupción afecta la provisión de bienes públicos únicamente cuando ésta cruza determinado umbral, por lo que la relación toma forma de "U". Al respecto, Petrou y Thanos (2014) estiman una relación corrupción-IED con esta misma forma, lo que significa que los efectos negativos se sienten cuando los niveles de corrupción son bajos o moderados, y los efectos positivos aparecen con altos estándares de corrupción.

Por otra parte, de acuerdo con la argumentación de Cieslik y Goczek (2018), ningún país está libre de corrupción, lo que se asocia con la idea de corrupción universal sin distinción de fronteras o modelos socioeconómicos (Popova \& Podolyakina, 2014). No obstante, la evidencia sugiere que este fenómeno es desenfrenado y más frecuente en el mundo en desarrollo (Svensson, 2005). En particular, América Latina (AL) y China se encuentran entre las regiones con mayor corrupción.

En los últimos años América Latina ha sufrido numerosos escándalos de corrupción, incrementando tanto el malestar social como los procesamientos de funcionarios gubernamentales y altos ejecutivos (Casas-Zamora \& Carter, 2017). Por su parte, en China, donde la separación entre el partido político gobernante y el Estado es mínima, la corrupción tiende a ser más fuerte y arraigada. Al respecto, Pesqué-Cela (2018) afirma que la corrupción, en distintas formas como soborno generalizado, compra de cargos públicos, malversación de fondos, impuestos ilegales, nepotismo y amiguismo, dentro del Partido Comunista se ha contagiado a la judicatura, la policía y las finanzas públicas, intensificándose desde el periodo de la reforma económica. El elevado nivel de corrupción en China ha motivado que distintos estudios académicos sostengan la existencia de corrupción implícita y explícita, ambas originadas en el sistema político (Yao, 2002).

En cualquier caso, tal como en América Latina, la corrupción motivó los esfuerzos anticorrupción llevando al arresto de 1.3 millones de funcionarios desde 2014, si bien únicamente el 3\% fue procesado penalmente (PesquéCela, 2018).

Estos problemas se reflejan en elevados índices de corrupción. Según datos de Transparencia Internacional (Transparency International, 2020), América Latina registró un índice de percepción de la corrupción (IPC) de 36.5 puntos en promedio en 2019; mientras que el índice en China fue de 41; en ambos casos por debajo del promedio global de 43. En contraste con el año 2001, salvo China y Uruguay, todos los países empeoraron su posición en el 
ranking mundial y nueve países de América Latina sufrieron un aumento en la percepción de corrupción (disminución del IPC).

Aparte, si se consideran los datos del desarrollo económico, medido a través del índice de desarrollo humano (IDH), se aprecia que los países latinoamericanos y asiáticos registran niveles catalogados en un rango medio a bajo. En 2019 el IDH fue de 74.3 en promedio para América Latina, y de 76 para China. Estos valores están por arriba a los de 2001, cuando se registró un promedio en América Latina de 66.8 y en China de 60. Si bien todos los países mejoraron en el IDH, aquéllos con los menores IPC (mayor corrupción) son simultáneamente los de menor desarrollo humano en 2019. Aún más, los países que registran mayores avances respecto a la corrupción percibida (mayores tasas de crecimiento del IPC) también reflejan las más altas tasas de crecimiento del IDH y viceversa. Los casos concretos de China, Brasil y México, los países más grandes en términos de PIB y población, confirman lo anterior.

De este modo, parece existir una relación entre corrupción y bienestar. $\mathrm{Al}$ respecto, tanto en la academia como en las discusiones políticas y sociales una afirmación común es que la corrupción afecta negativamente el desarrollo económico. Esto es, los bajos niveles de desarrollo económico pueden explicarse, al menos en parte, por el desempeño corrupto — reflejado en el IPCdel sistema socioeconómico en estos países, convirtiéndose en un obstáculo principal para el desarrollo.

En otros términos, la corrupción es una plaga para los países que desgasta el crecimiento y desarrollo humano de su población. Por ejemplo, Akhter (2004) argumenta que, en un contexto de globalización, la reducción en los niveles de corrupción mejora el desarrollo humano. Alves, Costa y da Dias (2017) señalan que la corrupción se vincula de manera íntima con el desarrollo humano, puesto que entornos corruptos propician el desvío de recursos originalmente destinados a aspectos sociales como salud, educación e infraestructura, impactando directamente en el IDH. Asimismo, argumentan que el desarrollo humano se relaciona con la libertad que disfruta cada persona, la cual, a su vez, se vincula con el acceso y calidad de los bienes públicos de una sociedad, dimensiones sujetas, al menos parcialmente, a actos de corrupción que tienden a socavarla.

La literatura para el caso concreto de América Latina y Asia es limitada y tiende a enfocarse en los efectos sobre variables macroeconómicas. Por ejemplo, Lucic et al. (2016), considerando un conjunto de 40 países desarrollados y en desarrollo, hallan un efecto negativo de la corrupción sobre el PIB 
de hasta por 10 años. Asimismo, siguiendo una estimación por el método generalizado de momentos (GMM) para un grupo de 60 países, incluyendo latinoamericanos y asiáticos, Ahmad, Aman, y Arfeen (2012) encuentran que reducciones de la corrupción afectan la tasa de crecimiento del PIB per cápita en forma de "U" invertida.

Otros efectos también se encuentran en variables como inflación (Özsahin \& Üçler, 2017); inversión privada (Everhart, Martínez \& McNab, 2003); exportaciones (Charoensukmongkol \& Sexton, 2011); empleo (Beltrán, 2016); emprendurismo (Colonnelli \& Prem, 2017) de manera negativa; inversión extranjera directa, de forma positiva particularmente para el caso de Asia (Talvitie, 2017); y gasto público en ambas direcciones, dependiendo de la forma precisa de la corrupción (Wong, 2016).

Asimismo, se estiman efectos negativos en variables del desarrollo en Latinoamérica y Asia. Kar y Saha (2012) identifican que la corrupción aumenta la desigualdad, medida por el coeficiente de Gini; Carballo (2010) establece que mayor corrupción se traduce en pobreza. De manera particular, Ackay (2006) considerando un conjunto de 63 países, entre ellos los estudiados en este documento, usa tres distintos índices de corrupción y estima, por medio de mínimos cuadrados ordinarios, que los países más corruptos tienden a mostrar menores niveles de desarrollo humano.

Así, se plantea la hipótesis de que si existe corrupción, medida por el índice de percepción de la corrupción, entonces el desarrollo económico, aproximado por el enfoque de desarrollo humano, es limitado. En última instancia, el desarrollo se trata de mejoras constantes en el bienestar humano, cuya sustentabilidad se ve amenazada por la magnitud y frecuencia de los actos de corrupción. El artículo tiene como objetivo determinar el efecto de la corrupción en el desarrollo humano en un grupo de 18 países latinoamericanos y en China. El periodo de estudio comprende los años 2001 a 2019. Se sigue una metodología econométrica de datos de panel.

El documento se estructura, además de la introducción, en cinco secciones. En la siguiente se revisa la literatura asociada con corrupción y desarrollo y se identifican algunas regularidades empíricas en esta temática en ambas regiones. Después se muestra brevemente la situación actual; seguida de la descripción de la metodología y los datos. Más tarde se estima el efecto de la corrupción en el IDH. Finalmente se presentan algunas reflexiones e implicaciones de política. 


\section{Corrupción y desarrollo económico}

Para las Naciones Unidas (United Nations, 2004) la corrupción es una plaga con amplios efectos corrosivos en las sociedades. Aunque no existe un acuerdo en la literatura respecto a la definición del fenómeno de la corrupción, diversas instituciones la definen como "el abuso del poder público para beneficios privados" (World Bank, 1997) y "mal uso, activo o pasivo, de los poderes de los funcionarios públicos (designados o elegidos) para obtener beneficios financieros privados o de otro tipo" (Organización para la Cooperación y el Desarrollo Económico [OCDE], 2013). La organización Transparencia Internacional lo define como el "abuso del poder confinado para ganancias privadas, financieras o no" (Transparency International, 2020).

Por su parte, el desarrollo humano se define como "la expansión de oportunidades que tienen las personas para llevar vidas que valoran" (Programa de las Naciones Unidas para el Desarrollo [PNUD], 2016), que se alcanza mediante la creación de capacidades humanas a través del desarrollo de recursos humanos — salud, nutrición, educación, capacitación, etcétera-. El índice de desarrollo humano (IDH) - o enfoque de desarrollo humano- se refiere a la expansión de la riqueza de la vida humana, en lugar de simplemente la riqueza de la economía de una sociedad. Por ende, se centra en mejorar la vida de las personas sin asumir que el crecimiento económico conduce automáticamente al aumento del bienestar de todas las personas. El IDH es un índice compuesto que incorpora tres dimensiones básicas del desarrollo humano: vivir una vida larga y saludable, medida por la esperanza de vida al nacer; habilidad de adquirir conocimientos, medida por los años promedio de escolaridad y la esperanza de años de escolaridad; y la habilidad de alcanzar estándares de vida decentes, medida por el ingreso nacional bruto per cápita. ${ }^{4}$

$\mathrm{Al}$ respecto, existen diversas razones empíricas por las que el desarrollo humano puede ser afectado por la corrupción. Así, además de producir una disminución del crecimiento económico y de los incentivos a la inversión,

4. El Programa de las Naciones Unidas para el Desarrollo (PNUD) (2019) clasifica en cuatro grupos a los países en función del IDH; esto es, desarrollo humano muy alto con IDH $\geq 0.800$, desarrollo humano alto con $0.700<\mathrm{IDH}<0.799$, desarrollo humano medio con $0.550<\mathrm{IDH}<0.699 \mathrm{y}$ desarrollo humano bajo con un IDH $<0.550$. 
el efecto se siente en los niveles de pobreza y desigualdad. ${ }^{5}$ Rose-Ackerman (2008) argumenta que la corrupción afecta la asignación de los beneficios económicos provocando una desigual distribución del ingreso dentro de un país. Para Lash (2004) la reducción de la eficiencia económica a raíz de la corrupción genera que la desigualdad aumente. Aún más, Akcay (2006) advierte que parte de la riqueza de un país se distribuye entre los agentes involucrados en actos de corrupción, contribuyendo a la desigualdad. Esto es de particular interés para los países analizados, puesto que la desigualdad ha aumentado en mayor proporción en economías con mayores niveles de corrupción. En este sentido, Aghion, Akcigit, Cagé y Kerr (2016) sostienen que reducir la corrupción tiene el mayor impacto potencial para incrementar el bienestar social, a través de un uso de los impuestos cercano al óptimo social.

Asimismo, para Justesen y Bjornskov (2014) los pobres son quienes más sufren por actos de corrupción, puesto que dependen en gran medida de los servicios prestados por los gobiernos y frecuentemente tienen que pagar sobornos para acceder a bienes y servicios públicos. La conexión entre corrupción, pobreza e IDH es clara. Los pobres, caracterizados por menor nivel de desarrollo humano, sufren de la carencia de estos bienes y servicios $y$, para acceder a ellos, deben enfrentar la corrupción que limita la cantidad y calidad de su disfrute.

Esto se asocia con la idea de Frisch (1996), quien señala que la corrupción es causa y efecto del subdesarrollo, siendo culpable de exacerbar la pobreza como una de sus principales manifestaciones. Lo anterior refleja la realidad de los países estudiados y enciende focos de alerta puesto que, para Blackburn y Forgues-Puccio (2010), la corrupción y pobreza pueden coexistir permanentemente, perpetuando el círculo perverso entre éstos.

Parte de la literatura es más concreta y analiza la relación de la corrupción con el desarrollo humano. Por ejemplo, Tran (2008) afirma que existe una relación de equilibrios múltiples entre estas dos dimensiones. El equilibrio virtuoso existe en los países ricos y el equilibrio perverso en los países pobres, lo que limita su potencial de desarrollo. Agrega que si el nivel de ingreso es

5. Balboa y Medalla (2006) identifican efectos principales de un régimen corrupto: i) proyectos de infraestructura sesgados en contra de los pobres, ya que los funcionarios públicos diseñan proyectos públicos que maximizarán los sobornos recibidos y minimizan las posibilidades de detección; ii) mayores cargas tributarias y menos servicios, afectando el ingreso disponible y el acceso a servicios de salud, educación, recreación, etc.; iii) menores oportunidades para la comercialización de productos de los agricultores y de creación y expansión de pequeñas y medianas empresas. 
muy bajo, el efecto de la corrupción es desenfrenado y el proceso de desarrollo humano se compromete. Esta idea es compartida por Pilapitiya (2004), para quien los regímenes corruptos amenazan los derechos humanos básicos y las libertades. Asimismo, Popova y Podolyakina (2014) afirman que este fenómeno tiende a afectar los sistemas de salud, educación e innovación, convirtiéndose en un peligro para cualquier país independientemente de su modelo social, puesto que impacta directamente en el desarrollo de capital humano.

Una consecuencia de la corrupción en términos sociales es que asigna de forma desigual las prestaciones de salud y retrasa su acceso, al tiempo que debilita la provisión de servicios públicos básicos. Como en lo general son las personas con menor IDH quienes más sufren este fenómeno, se tiene que la corrupción socava la red de seguridad social e incluso puede disuadir a los pobres de buscar derechos básicos y otros servicios públicos, quedando fuera de programas sociales, lo que restringe en última instancia el IDH.

En específico, la literatura encuentra que la corrupción influye en los sistemas públicos de salud y educación. Lewis (2006) argumenta que la provisión de servicios de salud depende de la combinación eficiente y oportuna de recursos financieros, humanos y suministros. Sin embargo, afirma que el alcance y naturaleza de la corrupción de los funcionarios públicos (nepotismo, sobornos, etc.), así como las irregularidades en compras públicas y la gestión del combate de la corrupción han minado la eficiencia de estos servicios. Para du Plessis (2014), el efecto de la corrupción en el sistema educativo se mantiene en el largo plazo en la actividad económica y en las relaciones sociales. En particular, señala que sus consecuencias se sienten con mayor fuerza en los pobres que, sin acceso a la educación o sin alternativas de alta calidad, tienen pocas posibilidades de escapar de la pobreza manteniendo los bajos índices de desarrollo humano.

Los estudios para América Latina y China que consideran el efecto de la corrupción en el desarrollo humano son escasos, si bien tienden a coincidir sobre los efectos de este fenómeno. Entre los documentos que encuentran que la corrupción daña el desarrollo medido por el IDH están Ackay (2006), Carballo (2010), López (2019), y Hoinaru, Buda, Borlea, Văidean y Achim (2020).

\section{Situación actual}

La Organización para la Cooperación y el Desarrollo Económico (OCDE) estima que al menos el 5\% del PIB mundial se gasta cada año en la corrupción, 
pagando cerca de un billón de dólares en sobornos y 2.6 billones en otras formas de corrupción. Las Naciones Unidas reconocen que ésta es un lastre para el cumplimiento de la Agenda 2030 para el Desarrollo Sostenible, con los países de América Latina entre los más corruptos y con fuertes problemas de pobreza y desarrollo.

En particular, para países como México o Brasil, representa entre el 5 y 9\% del PIB; en comparación, para China este valor es cercano al $4 \%$ del PIB. Históricamente, América Latina y China se han caracterizado por tener un débil sistema democrático y gobiernos autoritarios, una posible combinación para la expansión y arraigo de este fenómeno. Si bien se percibe que esta situación ha cambiado gradualmente en ambas regiones en las últimas tres décadas. A pesar de ello, el fenómeno permanece profundamente arraigado en las raíces de la sociedad, probando la complejidad para combatirse. Algunas estimaciones señalan que a pesar de que el número total de casos de corrupción ha disminuido, la cantidad de dinero involucrada en la corrupción tiende a aumentar (Yong, 2008).

Como ejemplo, se tienen los reconocidos casos de: "Papeles de Panamá" que involucra la publicación de 11 millones de documentos relacionados con más de 200 mil paraísos fiscales utilizados por personajes multimillonarios, entre ellos 143 políticos y 12 jefes de Estado de la región latinoamericana. "Papeles del Paraíso", similar al anterior con 13.4 millones de archivos vinculados a empresas multinacionales, políticos, jefes de Estado, entre otros. "Odebrecht", empresa constructora brasileña con presencia en 27 países de América Latina y África, envuelta en actos de corrupción en 11 países. Se estima que la gigante brasileña pagó poco más de 350 millones de dólares en distintos países latinoamericanos y recibió a cambio beneficios por más de tres mil millones dólares.

En el caso de la nación asiática no se han evidenciado casos de corrupción multimillonarios a escala internacional, pero el número de casos continúa siendo alto con efectos principalmente en la confianza sobre el Partido Comunista (PC). Esta situación cobra mayor peso al reconocer que el problema de corrupción en China se halla en la interacción entre las clases política y empresarial, donde la intervención gubernamental es el eje rector de la 
economía. ${ }^{6}$ Sobresalen los escándalos mediáticos y políticos de "Bon Xilai" y "Liu Han". El primero, ex ministro de comercio y jefe del PC en la ciudad de Chongqing, fue destituido del cargo y condenado a cadena perpetua por delitos de corrupción, abusos de poder y malversación de fondos públicos. El segundo, propietario de la mayor empresa privada en el sector minero, fue ejecutado por ser culpable de más de una decena de delitos de corrupción y homicidio (organizar, dirigir y participar en una organización criminal, hasta planear homicidios o vender armas ilegalmente).

En concreto, en la tabla 1 se muestra el IPC para América Latina y China durante 2001-2019. ${ }^{7}$ El promedio para América Latina pasó de 35.2 a 36.7 en el último año, por debajo del índice chino en esos mismos años. De cualquier forma, se observa una dinámica positiva en ambas regiones. No obstante, a pesar de los esfuerzos por combatir la corrupción, la evolución en la mayoría de estos países ha sido con lentitud. Por tanto, dado que el IPC refleja la percepción de expertos y empresarios de la corrupción en el sector público, cabe esperar que, en promedio, las condiciones para que se reproduzca este fenómeno en la realidad se modificaran ligeramente.

6. De acuerdo con el reporte de la revista Hurun —el Forbes chino-, desde hace dos décadas se han abierto investigaciones contra 35 de los casi tres mil millonarios en ese país (1\% del total). $\mathrm{Al}$ respecto, el número de billonarios pasó de 15 a 250 en sólo seis años (Chao, 2013), pero 17\% de éstos están siendo investigados por corrupción, cumpliendo condenas o han sido ejecutados (Rosen, 2012).

7. El índice clasifica 180 países y territorios según sus niveles percibidos de corrupción en el sector público de acuerdo con expertos y empresarios, usa una escala de 0 a 100, donde 0 es altamente corrupto y 100 es muy limpio (transparencia elevada). En todo caso, se puede considerar a los países por arriba de la media como los menos corruptos y aquéllos por debajo como los que enfrentan mayores problemas de corrupción. 


\section{Tabla 1}

Índice de percepción de la corrupción en América Latina

y China, países seleccionados

\begin{tabular}{|c|c|c|c|c|c|c|c|c|c|c|c|c|c|c|c|c|c|c|c|c|c|c|}
\hline Pais & 2001 & 2002 & 2003 & 2004 & 2005 & 2006 & 2007 & 2008 & 2009 & 2010 & 2011 & 2012 & 2013 & 2014 & 2015 & 2016 & 2017 & 2018 & 2019 & Crec & Pos01 & Pos19 \\
\hline Argentina & 35 & 28 & 25 & 25 & 28 & 29 & 29 & 29 & 29 & 29 & 30 & 35 & 34 & 34 & 32 & 36 & 39 & 40 & 45 & 1.41 & {$[10]$} & {$[4]$} \\
\hline Bolivia & 20 & 22 & 23 & 22 & 25 & 27 & 29 & 30 & 27 & 28 & 28 & 34 & 34 & 35 & 34 & 33 & 33 & 29 & 31 & 2.46 & [18] & [12] \\
\hline Brasil & 40 & 40 & 39 & 39 & 37 & 33 & 35 & 35 & 37 & 37 & 38 & 43 & 42 & 43 & 38 & 40 & 37 & 35 & 35 & -0.74 & [5] & [10] \\
\hline Chile & 75 & 75 & 74 & 74 & 73 & 73 & 70 & 69 & 67 & 72 & 72 & 72 & 71 & 73 & 70 & 66 & 67 & 67 & 67 & -0.62 & [1] & [2] \\
\hline China & 35 & 35 & 34 & 34 & 32 & 33 & 35 & 36 & 36 & 35 & 36 & 39 & 40 & 36 & 37 & 40 & 41 & 39 & 41 & 0.88 & [11] & [5] \\
\hline Colombia & 38 & 36 & 37 & 38 & 40 & 39 & 38 & 38 & 37 & 35 & 35 & 36 & 36 & 37 & 37 & 37 & 37 & 36 & 37 & -0.15 & [6] & [7] \\
\hline Costa Rica & 45 & 45 & 43 & 49 & 42 & 41 & 50 & 51 & 53 & 53 & 48 & 54 & 53 & 54 & 55 & 58 & 59 & 56 & 56 & 1.22 & [3] & [3] \\
\hline Ecuador & 31 & 35 & 33 & 29 & 30 & 28 & 30 & 30 & 30 & 30 & 26 & 32 & 29 & 32 & 33 & 31 & 32 & 34 & 38 & 1.14 & [12] & [6] \\
\hline El Salvador & 23 & 22 & 22 & 24 & 25 & 23 & 21 & 20 & 22 & 25 & 27 & 32 & 35 & 33 & 32 & 31 & 33 & 35 & 34 & 2.20 & [17] & [11] \\
\hline Guatemala & 36 & 34 & 37 & 42 & 42 & 40 & 40 & 39 & 34 & 36 & 34 & 38 & 38 & 39 & 39 & 36 & 28 & 27 & 26 & -1.79 & [9] & [16] \\
\hline Honduras & 29 & 25 & 24 & 22 & 25 & 26 & 28 & 31 & 34 & 32 & 27 & 33 & 29 & 32 & 28 & 28 & 29 & 29 & 26 & -0.60 & [13] & {$[17]$} \\
\hline México & 27 & 27 & 23 & 23 & 26 & 25 & 25 & 26 & 25 & 24 & 26 & 28 & 26 & 29 & 31 & 30 & 29 & 28 & 29 & 0.40 & [15] & [13] \\
\hline Nicaragua & 37 & 36 & 36 & 36 & 35 & 33 & 35 & 36 & 33 & 31 & 30 & 34 & 34 & 35 & 35 & 30 & 26 & 25 & 22 & -2.85 & [7] & {$[18]$} \\
\hline Panamá & 24 & 25 & 26 & 27 & 26 & 26 & 26 & 25 & 25 & 25 & 25 & 29 & 28 & 28 & 27 & 26 & 37 & 37 & 36 & 2.28 & [16] & [8] \\
\hline Paraguay & 37 & 30 & 34 & 37 & 35 & 31 & 32 & 34 & 34 & 36 & 33 & 38 & 35 & 37 & 39 & 38 & 29 & 29 & 28 & -1.54 & [8] & [14] \\
\hline Perú & 17 & 17 & 16 & 19 & 21 & 26 & 24 & 24 & 21 & 22 & 22 & 25 & 24 & 24 & 27 & 30 & 37 & 35 & 36 & 4.26 & [19] & [9] \\
\hline República Dominicana & 41 & 40 & 37 & 35 & 35 & 33 & 35 & 36 & 37 & 35 & 34 & 38 & 38 & 38 & 36 & 31 & 29 & 30 & 28 & -2.10 & [4] & [15] \\
\hline Uruguay & 51 & 51 & 55 & 62 & 59 & 64 & 67 & 69 & 67 & 69 & 70 & 72 & 73 & 73 & 74 & 71 & 70 & 70 & 71 & 1.86 & [2] & [1] \\
\hline Venezuela & 28 & 25 & 24 & 23 & 23 & 23 & 20 & 19 & 19 & 20 & 19 & 19 & 20 & 19 & 17 & 17 & 18 & 18 & 16 & -3.06 & [14] & {$[19]$} \\
\hline Promedio AL & 35.22 & 34.06 & 33.78 & 34.78 & 34.83 & 34.44 & 35.22 & 35.61 & 35.06 & 35.50 & 34.60 & 38.44 & 37.72 & 38.61 & 38.00 & 37.17 & 37.17 & 36.67 & 36.72 & 0.232 & 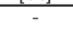 & 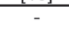 \\
\hline Promedio Total & 35.21 & 34.11 & 33.79 & 34.74 & 34.68 & 34.37 & 35.21 & 35.63 & 35.11 & 35.47 & 34.69 & 38.47 & 37.84 & 38.47 & 37.95 & 37.32 & 37.37 & 36.79 & 36.48 & 0.198 & - & - \\
\hline
\end{tabular}

Notas: Crec = crecimiento promedio anual; Pos01 = posición entre los países considerados en el año 2001; Pos19 = posición entre los países considerados en el año 2019.

Fuente: elaboración propia con datos de Transparency International (2020), disponible en: https://www.transparency.org/en/cpi 
En el primer año, el país con el mejor IPC es Chile (75), seguido por Uruguay (51) y Costa Rica (45). Para 2019, el país mejor posicionado es Uruguay (71), escoltado por Chile (67) y Costa Rica (56). En el otro extremo, Perú ocupa la última posición en 2001 y Venezuela en 2019. Países como México y Colombia prácticamente mantuvieron su posición relativa; el primero con un desempeño por debajo del promedio en cada año del periodo. China pasó de un IPC en 2001 de 35 a 41 en 2019, escalando seis lugares en el ranking. Asimismo, se observa un comportamiento oscilante de la percepción de la corrupción en las dos regiones, siendo 2012 y 2014 los años donde se alcanza el mayor IPC promedio, y 2003 con el menor IPC promedio (33.78).

Aparte, la tabla 2 muestra el IDH para América Latina y China durante 2001-2019. En el último año, el promedio para América Latina fue de 0.74, cuando en 2001 alcanzó los 0.67. China, en comparación, pasó de un índice de 0.60 a 0.76 . Se observa una evolución positiva en ambas regiones, aunque con un mejor desempeño del país asiático. Puesto que el IDH recoge tres aspectos críticos del desarrollo humano, cabe esperar que, en promedio, las condiciones de vida de estos países hayan mejorado. 
Héctor Flores Márquez, Ana Lilia Valderrama Santibáñez y Omar Neme Castillo

\section{Tabla 2}

Índice de desarrollo humano en América Latina y China, países seleccionados

\begin{tabular}{|c|c|c|c|c|c|c|c|c|c|c|c|c|c|c|c|c|c|c|c|c|c|c|}
\hline País & 2001 & 2002 & 2003 & 2004 & 2005 & 2006 & 2007 & 2008 & 2009 & 2010 & 2011 & 2012 & 2013 & 2014 & 2015 & 2016 & 2017 & 2018 & 2019 & Crec & Pos01 & Pos19 \\
\hline Argentina & 0.78 & 0.78 & 0.78 & 0.79 & 0.79 & 0.8 & 0.8 & 0.81 & 0.81 & 0.82 & 0.83 & 0.82 & 0.83 & 0.83 & 0.83 & 0.83 & 0.83 & 0.83 & 0.83 & 0.35 & [1] & [2] \\
\hline Bolivia & 0.61 & 0.62 & 0.62 & 0.62 & 0.79 & 0.63 & 0.64 & 0.64 & 0.64 & 0.65 & 0.66 & 0.66 & 0.67 & 0.67 & 0.67 & 0.67 & 0.70 & 0.70 & 0.70 & 0.79 & [15] & [15] \\
\hline Brasil & 0.69 & 0.69 & 0.7 & 0.7 & 0.63 & 0.71 & 0.71 & 0.72 & 0.72 & 0.72 & 0.73 & 0.73 & 0.75 & 0.75 & 0.75 & 0.75 & 0.76 & 0.76 & 0.76 & 0.55 & [7] & [7] \\
\hline Chile & 0.77 & 0.77 & 0.78 & 0.78 & 0.7 & 0.8 & 0.8 & 0.81 & 0.81 & 0.82 & 0.83 & 0.83 & 0.84 & 0.84 & 0.85 & 0.85 & 0.84 & 0.85 & 0.85 & 0.53 & [2] & [1] \\
\hline China & 0.6 & 0.61 & 0.62 & 0.63 & 0.79 & 0.65 & 0.67 & 0.68 & 0.69 & 0.7 & 0.7 & 0.71 & 0.72 & 0.73 & 0.74 & 0.74 & 0.75 & 0.76 & 0.76 & 1.3 & [16] & [11] \\
\hline Colombia & 0.66 & 0.66 & 0.67 & 0.67 & 0.64 & 0.68 & 0.69 & 0.69 & 0.7 & 0.7 & 0.71 & 0.71 & 0.72 & 0.72 & 0.73 & 0.73 & 0.76 & 0.76 & 0.76 & 0.79 & [11] & [8] \\
\hline Costa Rica & 0.71 & 0.72 & 0.72 & 0.72 & 0.68 & 0.73 & 0.74 & 0.74 & 0.75 & 0.75 & 0.76 & 0.76 & 0.77 & 0.77 & 0.78 & 0.78 & 0.79 & 0.79 & 0.79 & 0.62 & [5] & [5] \\
\hline Ecuador & 0.66 & 0.67 & 0.67 & 0.67 & 0.73 & 0.68 & 0.69 & 0.69 & 0.7 & 0.7 & 0.71 & 0.71 & 0.71 & 0.72 & 0.72 & 0.72 & 0.76 & 0.76 & 0.76 & 0.77 & [12] & [10] \\
\hline El Salvador & 0.67 & 0.68 & 0.68 & 0.68 & 0.68 & 0.69 & 0.7 & 0.7 & 0.71 & 0.71 & 0.72 & 0.72 & 0.74 & 0.74 & 0.74 & 0.74 & 0.66 & 0.67 & 0.67 & -0.03 & [10] & [16] \\
\hline Guatemala & 0.62 & 0.62 & 0.62 & 0.63 & 0.69 & 0.65 & 0.65 & 0.66 & 0.66 & 0.67 & 0.67 & 0.67 & 0.68 & 0.68 & 0.68 & 0.68 & 0.65 & 0.65 & 0.65 & 0.27 & [14] & [18] \\
\hline Honduras & 0.55 & 0.56 & 0.56 & 0.56 & 0.64 & 0.58 & 0.59 & 0.6 & 0.6 & 0.61 & 0.62 & 0.61 & 0.61 & 0.64 & 0.64 & 0.64 & 0.62 & 0.62 & 0.62 & 0.69 & [19] & [19] \\
\hline México & 0.56 & 0.57 & 0.57 & 0.58 & 0.58 & 0.59 & 0.59 & 0.6 & 0.61 & 0.61 & 0.61 & 0.61 & 0.62 & 0.62 & 0.62 & 0.63 & 0.76 & 0.77 & 0.77 & 1.77 & [18] & [6] \\
\hline Nicaragua & 0.7 & 0.71 & 0.71 & 0.72 & 0.58 & 0.73 & 0.73 & 0.74 & 0.74 & 0.75 & 0.75 & 0.75 & 0.75 & 0.76 & 0.76 & 0.76 & 0.65 & 0.65 & 0.65 & -0.40 & [6] & [17] \\
\hline Panamá & 0.58 & 0.58 & 0.58 & 0.59 & 0.72 & 0.6 & 0.6 & 0.61 & 0.61 & 0.62 & 0.63 & 0.63 & 0.64 & 0.64 & 0.65 & 0.65 & 0.79 & 0.80 & 0.80 & 1.77 & [17] & [4] \\
\hline Paraguay & 0.72 & 0.73 & 0.73 & 0.74 & 0.59 & 0.74 & 0.75 & 0.75 & 0.75 & 0.76 & 0.76 & 0.77 & 0.78 & 0.78 & 0.79 & 0.79 & 0.72 & 0.72 & 0.72 & 0.03 & [4] & [14] \\
\hline Perú & 0.63 & 0.63 & 0.64 & 0.64 & 0.74 & 0.65 & 0.66 & 0.66 & 0.67 & 0.67 & 0.68 & 0.68 & 0.69 & 0.69 & 0.69 & 0.69 & 0.76 & 0.76 & 0.76 & 1.04 & [13] & [9] \\
\hline República Dominicana & 0.68 & 0.69 & 0.69 & 0.69 & 0.65 & 0.7 & 0.71 & 0.71 & 0.72 & 0.72 & 0.73 & 0.73 & 0.74 & 0.74 & 0.74 & 0.74 & 0.74 & 0.74 & 0.74 & 0.51 & [8] & [12] \\
\hline Uruguay & 0.75 & 0.75 & 0.75 & 0.76 & 0.7 & 0.76 & 0.77 & 0.77 & 0.78 & 0.78 & 0.78 & 0.79 & 0.79 & 0.79 & 0.79 & 0.8 & 0.81 & 0.81 & 0.81 & 0.41 & [3] & [3] \\
\hline Venezuela & 0.68 & 0.69 & 0.7 & 0.7 & 0.76 & 0.72 & 0.73 & 0.74 & 0.75 & 0.76 & 0.77 & 0.77 & 0.77 & 0.77 & 0.77 & 0.76 & 0.73 & 0.73 & 0.73 & 0.36 & [9] & [13] \\
\hline Promedio AL & 0.668 & 0.673 & 0.676 & 0.68 & 0.683 & 0.691 & 0.697 & 0.702 & 0.707 & 0.712 & 0.719 & 0.719 & 0.728 & 0.731 & 0.733 & 0.734 & 0.742 & 0.743 & 0.743 & 0.59 & - & - \\
\hline Promedio Total & 0.664 & 0.67 & 0.673 & 0.677 & 0.688 & 0.689 & 0.696 & 0.701 & 0.706 & 0.712 & 0.718 & 0.719 & 0.727 & 0.731 & 0.734 & 0.734 & 0.742 & 0.744 & 0.744 & 0.63 & - & - \\
\hline
\end{tabular}

Notas: Crec = crecimiento promedio anual; Pos01 = posición entre los países considerados en el año 2001; Pos19 = posición entre los países considerados en el año 2019.

Fuente: elaboración propia con datos del PNUD (2020), disponible en: http://hdr.undp.org/en/data 
Los países con IDH más elevados en 2001 son Argentina (0.78), Chile (0.77), Uruguay (0.75), Paraguay (0.72) y Costa Rica (0.71). Para 2019, dada la intervención de los gobiernos democráticos mediante políticas y programas sociales, con la excepción de Paraguay, estos mismos países continúan en el top 5. Se presenta un ligero cambio en la cima, por lo que el mejor posicionado es Chile (0.85), seguido por Argentina (0.83), Uruguay (0.81), Panamá (0.80) y Costa Rica (0.79). En el otro extremo, Honduras ocupa la última posición en ambos años. México avanzó notablemente en el ranking de países, pasando al lugar 6; Brasil mantuvo su posición relativa, mientras que China pasó del lugar 16 entre este grupo de países al puesto 11 en 2019, subiendo cinco lugares en el ranking, un comportamiento similar el del IPC.

Se observa una tendencia positiva del desarrollo humano en las dos regiones, siendo los últimos cuatro años cuando se alcanza el mayor IDH y 2001 el de menor IDH; asimismo, considerado el promedio, la región mostró un comportamiento monótono creciente. Destacan China y México como los únicos países que no tuvieron retrocesos en ningún año.

Al considerar la dinámica conjunta de los dos índices, se identifican algunos aspectos de interés: i) en general, el IDH muestra mejor desempeño que el IPC; ii) la variación en el IPC es mayor entre países que la del IDH; iii) China es la única economía "ganadora" en ambos índices; iv) Venezuela muestra mejor desempeño en el IDH frente al IPC; v) Chile es "ganador" en IDH pero "perdedor" en corrupción; vi) grandes economías como México o Brasil registran una evolución media en las dos variables; vii) parece existir un comportamiento paralelo entre ambas variables. Esto último puede observarse en la figura 1. La tendencia positiva en la relación sugiere que a mayor IPC mayor el desarrollo humano. Esto es, el bienestar (IDH) es inversamente proporcional a la corrupción percibida (mayores IPC), comprobándose desde este enfoque la hipótesis planteada. 


\section{Figura 1}

IDH vis-á-vis IPC (dispersión)

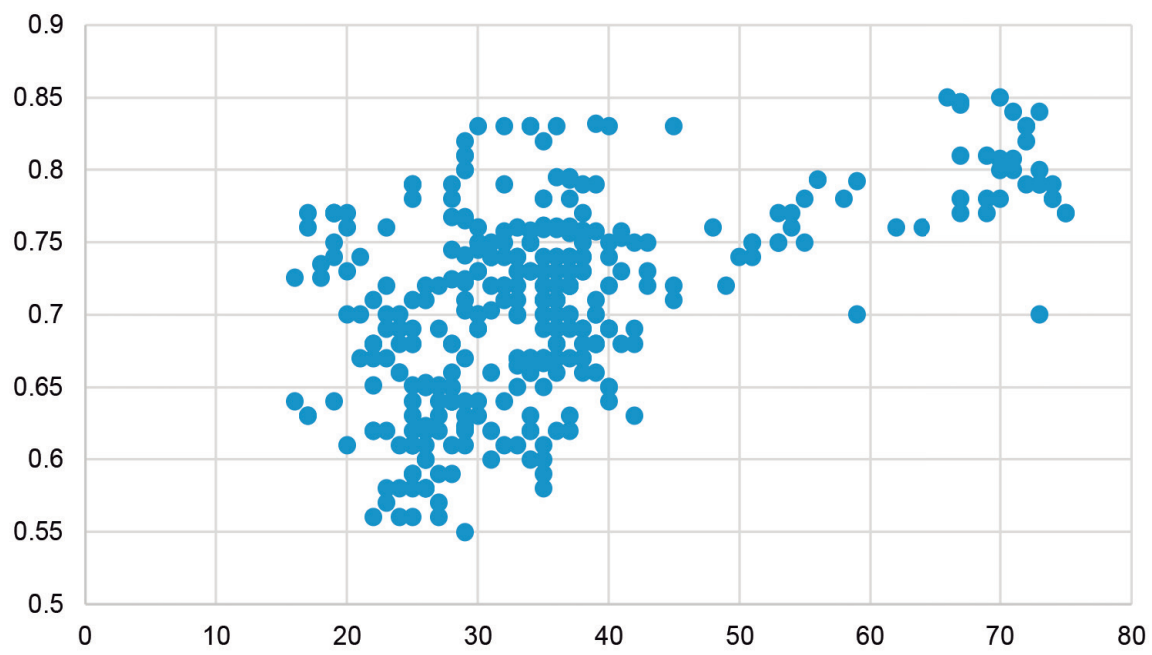

Fuente: elaboración propia con datos de Transparency International (2020), disponible en: https://www.transparency.org/en/cpi; y PNUD (2020), disponible en: http://hdr.undp.org/ en/data

\section{Metodología y datos}

La corrupción es un fenómeno socioeconómico difícil de medir. En general, la literatura emplea métodos comparativos para identificar regularidades entre los países. Se ha dado especial importancia a las percepciones que las personas tienen de la corrupción, discriminando entre sectores sociales, económicos y políticos. Entre las distintas opciones que miden la corrupción, en esta investigación se emplea el índice de percepción de corrupción (IPC), publicado por Transparencia Internacional, como variable proxy de la corrupción ya que incorpora una serie de datos de diferentes fuentes que proporcionan percepciones de empresarios y expertos del nivel de la corrupción en el sector público.

La literatura encuentra evidencia de la importancia de las instituciones políticas en la determinación del nivel de corrupción (Lederman, Loayza \& Soares, 2005); al mismo tiempo afirma que una de las causas de la corrupción en los países en desarrollo, es que la policía y los jueces tienden a ser focos de corrupción (Deysine, 1980). Bajo este argumento se incorporan al análisis 
los índices de Estado de derecho (ED) y eficiencia regulatoria (ER), que en su construcción consideran mediciones sobre los derechos de propiedad ${ }^{8} \mathrm{y}$ efectividad judicial ${ }^{9}$ el primero, y sobre libertades de negocios, ${ }^{10}$ laboral ${ }^{11} \mathrm{y}$ libertad monetaria, ${ }^{12}$ el segundo.

Algunas investigaciones empíricas han concluido que son causa de corrupción: políticas asociadas a grandes proyectos de inversión (debido a la discrecionalidad de las decisiones), la distribución de subsidios, la compra de bienes y servicios por el Estado y su provisión a precios inferiores a los de mercado (Tanzi, 1998). Para incorporar el papel del gobierno en el modelo se utiliza el índice de tamaño de gobierno (TG ${ }^{13}$ que capta la tasa impositiva, la sanidad financiera y el gasto de gobierno. Por último, se incorpora el índice de mercados abiertos (OM), puesto que se asocia a la corrupción con mercados poco competitivos y economías cerradas (Henderson, 1999). Se utiliza el PIB del año anterior para aproximar la dinámica económica de los países.

Como variable proxy del desarrollo económico se utiliza el IDH, ${ }^{14} \mathrm{creado}$ por el Programa de las Naciones Unidas para el Desarrollo (PNUD) para determinar el nivel de desarrollo de los países. Se espera que el Estado de derecho, la eficiencia regulatoria, la apertura comercial y el IPC tengan una relación

8. Evalúa la medida en que el marco legal de un país permite a las personas acumular libremente la propiedad privada, garantizada por leyes claras y efectivas. Mide el grado en que las leyes protegen los derechos de propiedad privada y la medida en que se respetan esas leyes. A mayor protección legal efectiva mayor el puntaje de un país (100) y viceversa (0).

9. El buen funcionamiento del marco jurídico es esencial para proteger los derechos ciudadanos frente a actos ilícitos cometidos por terceros (incluidos gobiernos y grupos de poder); lo que requiere sistemas judiciales eficientes y justos para garantizar el respeto de las leyes, y las medidas legales contra violaciones. A mayor efectividad judicial mayor el puntaje (100) y viceversa (0].

10. El puntaje está en el rango 0-100, donde 100 indica el entorno comercial más libre.

11. Considera distintos aspectos del marco legal y regulatorio del mercado laboral, como regulaciones sobre salarios mínimos, leyes que inhiben despidos, requisitos de indemnización y restricciones a la contratación y horas trabajadas. Toma el valor 0-100, donde 100 representa mayor libertad laboral.

12. Combina una medida de estabilidad de precios con una evaluación de los controles sobre éstos. Tanto la inflación como los controles de precios distorsionan la actividad del mercado. La estabilidad de precios sin intervención es el estado ideal para el mercado libre. Su escala es 0-100, donde 100 representa libertad monetaria plena.

13. El índice de tamaño de gobierno es un promedio ponderado entre sus subcomponentes (tasa impositiva, sanidad financiera y gasto de gobierno). En una escala de 0 a 100, un mayor puntaje representa un mayor tamaño de gobierno; de igual modo un porcentaje menor representa un menor tamaño de gobierno.

14. El IDH se ubica en el rango 0-1, siendo 0 el nivel más bajo y 1 el más alto. La PNUD clasifica a los países en tres grandes grupos: países con alto desarrollo humano con IDH > 0.8. Los países con desarrollo humano medio tienen un IDH entre $0.5,0.8$, y países con desarrollo humano bajo con IDH $<0.5$. 
positiva con el desarrollo económico; contrariamente, se espera una relación negativa del tamaño del gobierno con éste.

La información de las variables (ED, ER, TG, OM) se obtiene de la base de datos de la ONG Heritage (The Heritage Foundation, 2020). Los índices descritos son componentes del índice de libertad económica publicado por esta organización. Asimismo, el IDH corresponde a la base de datos del PNUD (2020) y el PIB, en dólares americanos de 2010, se toma de la base de datos del Banco Mundial (World Data Bank, 2020). Debido a la disponibilidad de datos para los 19 países seleccionados (tabla 1 ), el estudio considera el periodo 2001-2019.

Para el análisis formal se construye un modelo econométrico utilizando la técnica de datos panel. Este tipo de modelos generalmente muestran problemas de autocorrelación, heterocedasticidad, dependencia transversal y sesgo por multicolinealidad. Para garantizar resultados eficientes deben considerarse estos aspectos. Por lo que, en el análisis del efecto de la corrupción sobre el desarrollo económico, medido por el IDH, se utilizan las técnicas de datos panel estática en conjunto con la transformación de Prais-Winsten, y de datos de panel dinámica por el método generalizado de momentos (GMM) y su versión extendida conocida como sys-GMM.

Se emplea un modelo de datos de panel como metodología para estimar efectos significativos de la corrupción sobre el desarrollo económico en los países analizados. Esta metodología considera tanto su comportamiento en el tiempo como la existencia de heterogeneidades; asimismo, permite incrementar los grados de libertad en el tiempo y controlar por variables no observadas (Pignataro, 2018). Formalmente:

$I D H_{i t}=\alpha_{i}+\beta_{\mathbf{1}} I P C_{i t}+\beta_{\mathbf{2}} E D_{i t}+\beta_{\mathbf{3}} E R_{i t}+\beta_{\mathbf{4}} T G_{i t}+\beta_{\mathbf{5}} O M_{i t}+\beta_{\mathbf{6}} P I B_{i t-1}+u_{i t}$

Donde $i$ es la observación individual de cada país, $t$ el tiempo, PIB el Producto Interno Bruto e IPC, ED, ER, TG y OM, los índices de percepción de la corrupción, de Estado de derecho, de eficiencia regulatoria, de tamaño de gobierno, y de mercados abiertos, respectivamente. Los parámetros $\alpha_{i}, \beta_{1}$ a $\beta_{6}$ son los coeficientes a estimar y se esperan $\beta_{i}>0$.

El modelo de efectos fijos debe cumplir con una serie de condiciones para su consistencia y validez. En particular, se prueba que los datos no reflejen dependencia transversal, y no muestren heterocedasticidad, autocorrelación de primer orden, ni multicolinealidad. Se utilizan distintas pruebas que per- 
miten determinar la robustez de los errores estándar y, por tanto, el uso del modelo (Pesaran, Wald, Wooldridge, Modified DW, Baltagi-Wu-LBI y factor de inflación de la varianza - FIV-).

Para abordar estos aspectos se plantea una transformación del modelo que consiste en una regresión Prais-Winsten con errores estándares corregidos para paneles correlacionados (PCSE, por sus siglas en inglés), considerados robustos para alteraciones por heteroscedasticidad y autocorrelación (Greene, 2012). Este modelo reporta correctamente los coeficientes al ser consistentes (Blackwell, 2005), particularmente para periodos de tiempo muy grandes (Beck y Katz, 1995). Por el contrario, Hoechle (2007) comprueba que para bases con $\mathrm{T}<\mathrm{N}$ el estimador PCSE no tiene buen desempeño.

La transformación Prais-Winsten es un procedimiento de mínimos cuadrados generalizados. Usa datos cuasi diferenciados $Y_{i t}-\rho Y_{i t-1}, X_{i t}-\rho X_{i t-1}$ y una corrección $\left(1-\rho^{2}\right)^{(1 / 2)}$ para la primera observación. Este factor se necesita para que las perturbaciones transformadas sean homoscedásticas, pues $\sigma_{u}^{2}=\sigma_{\varepsilon}^{2}$ $\left(1-\rho^{2}\right) I_{t}$. Con esta transformación se mejora la eficiencia de la estimación en especial para muestras pequeñas (Wooldridge, 2002).

Como alternativa, se emplea el método generalizado de momentos (GMM) propuesto por Arellano y Bover (1995), que detalla un modelo dinámico con efectos específicos por país invariables en el tiempo. Esto parece plausible para el caso del IDH si se considera que otras variables fuera del análisis, como el régimen político, muestran sólo pequeñas variaciones en el tiempo. El modelo toma las primeras diferencias para eliminar los efectos invariables por país. La ecuación (1) se especifica como:

$I D H_{i t}-I D H_{i t-1}=\alpha+\beta_{1}\left(I D H_{i t-1}-I D H_{i t-2}\right)+\beta_{\mathbf{2}}\left(X_{i t}-X_{i t-1}\right)+\left(u_{i t}-u_{i t-1}\right)$

Para considerar la posible endogeneidad entre las variables explicativas, $X_{i t}, \mathrm{y}$ la variable dependiente, $I D H_{i t}$, (2) se estima usando los valores rezagados de las variables endógenas como instrumentos, los cuales son válidos si el término de error, $u_{i t}$, no está correlacionado serialmente, es decir, son independientes.

No obstante, existen algunas limitaciones estadísticas para una estimación directa de variables instrumentales con la metodología GMM. En concreto, los niveles rezagados hacen que los instrumentos sean débiles cuando se especifican en diferencias, principalmente en muestras pequeñas. AlonsoBorrego y Arellano (1996) establecen que el estimador GMM en primeras diferencias enfrenta sesgo de muestra finita y baja precisión. Como solución, 
Blundell y Bond (1998) proponen un modelo dinámico ampliado conocido como sistema-GMM (sys-GMM, por sus siglas en inglés), que combina las primeras diferencias rezagadas de la variable dependiente, $I D H_{i t}$, con sus niveles rezagados. Los instrumentos de la regresión en niveles son las diferencias rezagadas y los instrumentos para las regresiones en primeras diferencias son los niveles rezagados.

Un problema es que los instrumentos adicionales pueden no ser válidos (sobre identifican a las variables instrumentadas). Empero, Blundell, Bond y Windmeijer (2000) establecen que el sys-GMM tiene mejores propiedades de muestra finita en términos de sesgo. Para ello es necesario realizar, tanto para el GMM como para sys-GMM, la prueba de sobreidentificación de restricciones de Hansen, que se distribuye asintóticamente como una chi-cuadrada, y que es consistente en presencia de heterocedasticidad y autocorrelación.

Además, tal como el GMM, el modelo supone que el término de error no está correlacionado serialmente. La autocorrelación puede presentarse puesto que la variable dependiente rezagada también se asocia con los efectos específicos a nivel país. Es razonable aceptar cierto grado de persistencia en las condiciones que determinan el IDH de un determinado país. Para la consistencia del estimador se requiere que no exista correlación serial de segundo orden en los residuos diferenciados; por ende, se aplica la prueba de Arellano y Bond (1991) de autocorrelación.

Por último, para mantener los efectos individuales entre países se adicionan al modelo variables dicotómicas, por lo que se crean 19 variables artificiales con la especificación, $D_{i t}=1$ para el $i$-ésimo país y 0 si no lo es; siendo $i$ $=$ Argentina,Bolivia,...,Venezuela y $t=2001,2002, \ldots, 2019$.

\section{Resultados}

En la tabla 3 se resumen los resultados de las pruebas a la especificación de efectos fijos. Para corroborar el supuesto de efectos individuales entre países, se realizó la prueba de multiplicador de Lagrange de Breusch y Pagan (BPLM) diseñada para evaluar los efectos aleatorios. ${ }^{15}$ Los resultados indican que la prueba BPLM rechaza la hipótesis nula, es decir, hay efectos individuales en el modelo.

15. La hipótesis nula es que la varianza específica individual es cero, es decir $H_{0}:\left({ }_{\downarrow} \mathrm{u}^{\dagger} 2=0\right.$. 
Para descartar que estos efectos sean aleatorios, se llevó a cabo la prueba de Hausman en donde la hipótesis nula es que el modelo de efectos aleatorios es preferido al de efectos fijos. La prueba arrojó un resultado significativo, por lo que se afirma que el modelo de efectos fijos es mejor especificación.

\section{Tabla 3}

Pruebas realizadas al modelo de efectos fijos

\begin{tabular}{lc}
\hline & Pruebas \\
\hline BPLM & $863.970 *$ \\
Hausman & $85.550 *$ \\
$\quad$ Dependencia transversal & \\
Pesaran & 1.758 \\
$\quad$ Multicolinealidad & \\
VIF & 14.53 \\
$\quad$ Autocorrelación & \\
Modified DW & 5.77 \\
Baltagi-Wu-LBI & 1.1796 \\
$\quad$ Heterocedasticidad & \\
Wooldridge & $145.84 *$ \\
Wald & $185.080 *$ \\
\hline
\end{tabular}

Nota: ${ }^{*}$ significativos al $1 \%$.

Fuente: elaboración propia con base en las estimaciones de STATA.

Además, las pruebas sugieren que si bien los datos no muestran dependencia transversal, enfrentan niveles significativos de heterocedasticidad y autocorrelación de primer orden, combinados con multicolinealidad. ${ }^{16}$

Ante esto, el documento estima modelos de panel estáticos (PCSE) y dinámicos (GMM). Como se indicó, para mantener los efectos individuales entre países se incluyen variables dicotómicas; se examinó su pertenencia al

16. De acuerdo con el indicador VIF existe multicolinealidad. Una posibilidad de corrección es la eliminación de posibles variables generadoras. Se identifica, de acuerdo con la matriz de correlaciones, que la variable eficiencia regulatoria y el PIB muestran los mayores coeficientes. Sin embargo, al eliminarlos, los errores cuadrados medios (ECM) no disminuyen considerablemente, por lo que, dada la relevancia de considerar una variable económica y esa dimensión del índice de libertad económica, se decide mantenerlas en el modelo. 
modelo a través de una prueba $\mathrm{F}^{17}$ Se encontró que los regresores adicionales son estadísticamente diferentes de cero (tabla 4).

También en la tabla 4 se muestran los resultados de las estimaciones estáticas y dinámicas, con coeficientes diferenciados o comunes para el índice de corrupción. En general, se observa que los coeficientes de todas las variables tienden a ser sistemáticamente significativos a través de las especificaciones, salvo para las variables "Estado de derecho" en las estimaciones PCSE y "mercados abiertos" en los modelos PCSE y sys-GMM. Asimismo, los signos de los coeficientes tienden a permanecer y sus magnitudes se mantienen en el mismo rango. Esto contribuye a la robustez de los resultados y, por tanto, a aceptar las inferencias respecto al efecto de la corrupción sobre el desarrollo.

En particular, respecto a la corrupción los resultados encontrados son los esperados, esto es, el índice de percepción de la corrupción es positivo y estadísticamente significativo. En consecuencia, altos niveles en IDH se acompañan de altos niveles en IPC (menor percepción de corrupción). Así, alzas en los niveles de corrupción provocarían un efecto contrario en el desarrollo. Este hallazgo sugiere que la corrupción podría frenar el desarrollo en los países latinoamericanos. Posibles causas de este freno se asocian con los efectos negativos de la corrupción en variables económicas como finanzas públicas (Cooray et al., 2017), crecimiento económico (Cieslik \& Goczek, 2018), desigualdad en el ingreso (Rose-Ackerman, 2008), degradación de servicios públicos (Justesen \& Bjornskov, 2014), desarrollo de capital humano (Popova \& Podolyakina, 2014), entre otras.

El PIB muestra una relación positiva con el desarrollo, por lo que cabe esperar que economías más grandes y dinámicas tengan mayor desarrollo, como es el caso de China, Brasil y México que, a pesar de la elevada corrupción, alcanzan niveles de desarrollo relativamente altos. En resumen, el tamaño de la economía puede diluir parcialmente el efecto negativo de la corrupción en el desarrollo. Resultados como los de Ranis (2004), Teker y Güner (2016) y Bechtel (2018) también muestran una relación cercana entre PIB y desarrollo humano.

17. La hipótesis nula es que los coeficientes adicionales son iguales a cero, es decir $\beta_{0 i}$ es una intersección constante para todos los grupos de países $\left(H_{0}: \beta_{0 i}=\beta_{0}\right)$. El estadístico $\chi^{2}$ calculado es 9828.30 con $p$-value $=0.000$. 


\section{Tabla 4}

Resultados de las estimaciones

\begin{tabular}{|c|c|c|c|c|c|c|}
\hline Variable dependiente: idh & $\begin{array}{c}{[1]} \\
\text { Estimación PCSE }\end{array}$ & $\begin{array}{c}{[2]} \\
\text { Estimación PCSE } \\
\text { (coeficientes } \\
\text { diferenciados) }\end{array}$ & $\begin{array}{c}{[3]} \\
\text { Estimación GMM }\end{array}$ & $\begin{array}{c}4] \\
\text { Estimación GMM } \\
\text { (coeficientes } \\
\text { diferenciados) }\end{array}$ & $\begin{array}{c}\text { [5] } \\
\text { Estimación sys-GMM }\end{array}$ & $\begin{array}{c}6] \\
\text { Estimación Sys- } \\
\text { GMM (coeficientes } \\
\text { diferenciados) }\end{array}$ \\
\hline IDHR & - & - & - & - & $0.4219^{*}(0.007)$ & $1.1674^{*}(0.000)$ \\
\hline$P I B$ & $0.09361^{\star}(0.008)$ & $0.02936^{*}(0.000)$ & $0.1112^{\star \star}(0.046)$ & $0.12120^{*}(0.022)$ & $0.00490^{\star \star}(0.027)$ & $0.02714^{*}(0.000)$ \\
\hline$E D$ & $-0.00018^{\star \star \star}(0.038)$ & $0.00017(0.082)$ & $-0.00015^{*}(0.001)$ & $-0.00016^{* \star *}(0.036)$ & $-0.00036^{* * *}(0.042)$ & $\mathrm{di}$ \\
\hline$E R$ & $0.00007(0.082)$ & $0.00086^{*}(0.017)$ & $0.00188^{*}(0.034)$ & $0.00241^{\star \star}(0.018)$ & $0.0009^{* *}(0.028)$ & $0.0012^{*}(0.024)$ \\
\hline$T G$ & $-0.00034^{* * *}(0.027)$ & $-0.00096^{*}(0.003)$ & $-0.00029^{* * \star}(0.039)$ & $-0.00036^{* \star *}(0.045)$ & $-0.00063^{* * *}(0.048)$ & $-0.00021^{* * *}(0.009)$ \\
\hline$M A$ & $0.00047(0.094)$ & $0.00049^{* \star *}(0.031)$ & $0.00070^{* \star \star}(0.044)$ & $0.00059^{\star \star \star}(0.031)$ & $0.00048(0.059)$ & $0.0012(0.062)$ \\
\hline IPC & $0.00149 *(0.008)$ & - & $0.00305^{*}(0.000)$ & - & $0.00201^{*}(0.001)$ & - \\
\hline$c$ & $-1.7675^{\star}(0.000)$ & $-1.3809^{*}(0.000)$ & $-1.1340 *(0.000)$ & $-1.9901^{*}(0.000)$ & $0.3140^{* * *}(0.014)$ & $-1.4483^{*}(0.001)$ \\
\hline \multicolumn{7}{|l|}{ Dummies } \\
\hline Argentina & $0.0424^{\star}(0.002)$ & $0.0034^{\star}(0.000)$ & - & $0.0023^{\star \star \star}(0.016)$ & - & $0.0034^{*}(0.000)$ \\
\hline Bolivia & $0.1770^{*}(0.000)$ & $0.0019^{* *}(0.044)$ & - & $0.0019^{*}(0.001)$ & - & $0.0010 *(0.000)$ \\
\hline Brasil & $-0.2096^{*}(0.000)$ & $-0.0008^{* *}(0.028)$ & - & $-0.0018^{\star \star \star}(0.043)$ & - & $-0.0001^{* \star *}(0.044)$ \\
\hline Chile & $0.0450^{* * *}(C .025)$ & $0.0019^{*}(0.000)$ & - & $0.0039^{* *}(0.025)$ & - & $0.0024^{*}(0.000)$ \\
\hline China & $-0.3140 *(0.000)$ & $-0.0017^{*}(0.014)$ & - & $-0.0111^{*}(0.001)$ & - & $-0.0014^{*}(0.000)$ \\
\hline Colombia & $-0.0444^{* *}(0.050)$ & $0.0006^{* \star *}(0.041)$ & - & $0.0059^{* *}(0.046)$ & - & $0.0038^{\star \star \star}(0.042)$ \\
\hline Costa Rica & $0.1792^{*}(0.000)$ & $0.0026 *(0.000)$ & - & $0.0003^{*}(0.000)$ & - & $0.0022 *(0.000)$ \\
\hline Ecuador & $0.2195^{\star}(0.000)$ & $0.0037^{\star}(0.000)$ & - & $0.0038^{\star}(0.040)$ & - & $0.0042^{* * *}(0.037)$ \\
\hline El Salvador & $0.0938^{*}(0.001)$ & $0.0011^{* \star}(0.018)$ & - & $0.0025^{*}(0.008)$ & - & $0.0034^{\star \star \star}(0.011)$ \\
\hline Guatemala & $0.1422^{*}(0.000)$ & $0.0001(0.087)$ & - & $0.0014^{\star \star}(0.048)$ & - & $0.0009^{*}(0.000)$ \\
\hline Honduras & $-0.2301 *(0.000)$ & $-0.0036^{*}(0.001)$ & - & $-0.0026^{* *}(0.045)$ & - & $-0.0054^{\star \star \star}(0.041)$ \\
\hline México & $0.2034^{*}(0.000)$ & $0.0043^{*}(0.000)$ & - & $0.0033^{*}(0.019)$ & - & $0.0029 *(0.000)$ \\
\hline Nicaragua & $0.1285^{*}(0.001)$ & $0.0014^{* * *}(0.039)$ & - & $0.0110^{*}(0.000)$ & - & $0.0006^{*}(0.000)$ \\
\hline Panamá & $0.2246^{*}(0.000)$ & $0.0041^{*}(0.000)$ & - & $0.0090^{*}(0.000)$ & - & $0.0027^{*}(0.000)$ \\
\hline Paraguay & $0.0231^{* * *}(0.019)$ & $0.0013^{*}(0.009)$ & - & $0.0017^{*}(0.000)$ & - & $0.0006^{\star}(0.000)$ \\
\hline Perú & $0.1364^{\star}(0.000)$ & $0.0027^{\star}(0.000)$ & - & $0.0039^{\star \star}(0.030)$ & - & $0.0018^{*}(0.000)$ \\
\hline República Dominicana & $0.1037^{*}(0.000)$ & $0.0019^{*}(0.008)$ & - & $0.0026^{\star \star *}(0.012)$ & - & $0.0016^{\star}(0.000)$ \\
\hline Uruguay & $0.1794^{*}(0.000)$ & $0.0022^{*}(0.000)$ & - & $0.0032^{*}(0.000)$ & - & $0.0029^{*}(0.000)$ \\
\hline Venezuela & $0.0116^{* * *}(0.012)$ & $0.0019^{* *}(0.024)$ & - & $0.0015^{* * *}(0.022)$ & - & $0.0013^{\star \star \star}(0.026)$ \\
\hline$R^{2}$ & 0.778 & 0.996 & - & - & - & - \\
\hline No. de observaciones & 361 & 342 & - & - & - & - \\
\hline No. de instrumentos & - & - & 171 & 225 & 41 & 55 \\
\hline Tests Arellano-Bond para AR(1) $[\mathrm{Pr}>\mathrm{z}]$ & - & - & 0.009 & 0.0019 & 0.007 & 0.004 \\
\hline Tests Arellano-Bond para AR(2) $[\mathrm{Pr}>\mathrm{z}]$ & - & - & 0.050 & $0.2296^{*}$ & 0.051 & 0.886 \\
\hline Test de Sargan/Hansen de sobreidentificación & - & - & 0.988 & 0.946 & 0.985 & 0.999 \\
\hline
\end{tabular}

Notas: errores estándar en (). ${ }^{*},{ }^{* *}$ y ${ }^{* * *}$ Significancia al 1, 5 y 10\%, respectivamente. IDHR: índice de desarrollo humano rezagado un periodo.

Las variables dummies para las especificaciones 2,3 y 4 son dicotómicas interactivas.

Fuente: elaboración propia con base en las estimaciones de STATA. 
De igual forma, se estima un efecto positivo y significativo del Estado de derecho sobre el IDH. Este resultado es similar al de Haggard, MacIntyre y Tiede (2008), quienes señalan que garantizar derechos de propiedad y el cumplimiento de contratos apuntalan la inversión y el comercio que, a su vez, avivan el desarrollo económico. Esto significa que un Estado de derecho que propicie la protección y seguridad jurídica favorece el desarrollo, o, en palabras de Boettke y Subrick (2003), representa la estructura necesaria que promueve la prosperidad y las capacidades humanas.

Por tanto, Estados más fuertes en términos de garantizar las leyes, y aún más, en un sentido amplio, instituciones formales e informales, con reglas, valores y mecanismos alineados a las leyes representan condiciones básicas para el desarrollo económico y, en particular, favorecen el IDH. Países con instituciones confiables que actúen bajo la ley contribuyen a que nadie esté por arriba de la ley y, como resultado, una distribución más equitativa. En términos del Banco Mundial (2018), el respeto del Estado de derecho se asocia con mayores retornos del capital humano.

También se halla que la eficiencia regulatoria impacta en forma positiva (estadísticamente significativo) al desarrollo. Los resultados son consistentes con los de Jalilian, Kirkpatrick y Parker (2007), quienes establecen que tanto la eficiencia como la calidad de las regulaciones influyen en el desempeño económico. Haidar (2012) también establece que reformas en las regulaciones del mercado favorecen el crecimiento económico, que deriva en desarrollo. Así, gobiernos que establezcan regulaciones que promuevan la operación eficiente del mercado, que incluye control inflacionario y un marco regulatorio adecuado del trabajo, impulsan su desarrollo económico.

El tamaño del gobierno presenta una relación negativa, esto es, gobiernos con tasas impositivas y niveles de gasto más grandes propician menores niveles de desarrollo. Evidencia similar es presentada por Afonso y Tovar (2011) y Martins y Vega (2013). En particular, Davies (2009) argumenta que un excesivo tamaño del gobierno tiende a disminuir el IDH. Este efecto puede relacionarse con la corrupción, pues se asocia a gobiernos corruptos con volúmenes de gasto excesivos (en especial en infraestructura). Lo anterior está en línea con Aghion et al. (2016), por lo que emplear los impuestos de manera cercana al óptimo social, que implica menor corrupción, contribuye al bienestar social.

Por último, se encuentra una relación no significativa de la variable apertura de mercados en las estimaciones 1,5 y 6 . En las estimaciones 2 y 3 el 
coeficiente significativo muestra un signo positivo, sugiriendo que la mayor apertura comercial, atracción de inversiones extranjeras — productivas o financieras - se traduce en mayores niveles de desarrollo. Un efecto similar de esta variable sobre el IDH es confirmado por Davies y Quinlivan (2006) y por Yilmaz y Tag (2016). No obstante, este resultado debe tomarse con cautela y requiere de mayor evidencia.

Al contrastar los resultados con los datos, se observa que efectivamente los países que presentan un mayor nivel de desarrollo son los que alcanzan menores niveles de corrupción (véanse tablas 1 y 2). Los países con mayor desarrollo, medido por el IDH, son Chile, Argentina, Uruguay, Panamá y Costa Rica, que a su vez ocupan los primeros lugares en el IPC - excepto Argentina-. Venezuela y México registran un nivel de desarrollo mayor respecto a su posición en el IPC. Así, la sensibilidad y el tamaño de la economía son factores que pueden aminorar o acrecentar el efecto de la corrupción en el desarrollo. Aparte, se explora si los coeficientes de la variable IPC varían entre países, ${ }^{18}$ por lo que se consideran variables interactivas desagregadas por país para la variable de corrupción. Los resultados se muestran en la tabla 4, columnas 2 , 4 y 6 . Notablemente todos los coeficientes son significativos en las tres especificaciones, excepto para Guatemala en la estimación PCSE. Se aprecia que los efectos de la corrupción son diferentes entre las economías. Salvo para Brasil, China y Honduras, los signos de la variable IPC son positivos. Esto confirma el efecto de la corrupción cuando se estima para los países agrupados. No obstante, los coeficientes son de valores relativamente pequeños.

Considerando las tres especificaciones, los países con mayor sensibilidad a la corrupción son Ecuador, Panamá, Perú, México, Colombia y Argentina. En contraste, los países menos sensibles son Bolivia, República Dominicana, Guatemala, Paraguay y Venezuela. Estos resultados se mantienen al considerar exclusivamente los resultados sys-GMM.

Estos resultados sugieren que menor percepción de corrupción, reflejado en altos estándares del IPC, tienden a traducirse en mejores índices de desarrollo. Por tanto, el arraigado fenómeno de la corrupción en la sociedad representa un obstáculo para el desarrollo. Como se señaló, una consecuencia de la corrupción en términos sociales es que asigna de forma desigual las

18. Las variables dicotómicas interactivas se construyen al multiplicar cada variable dicotómica por la variable explicativa IPC. Formalmente, $D \_$interactiva ${ }_{i t}=D_{i t}{ }^{*} I P C_{i t}$. 
prestaciones de salud y retrasa su acceso, al tiempo que debilita la provisión de servicios públicos básicos.

Por el contrario, para Brasil, China y Honduras el coeficiente es negativo, sugiriendo que mejor calificación en el IPC, que implica menor corrupción percibida, entorpece el proceso de desarrollo. Esto se alinea con el planteamiento de Ahmad et al. (2012) sobre la existencia de un nivel distinto de cero de la corrupción como maximizador del crecimiento. Aún más, para el caso del país asiático, las estimaciones confirman la idea de la "paradoja de la corrupción", en la que altos niveles de este fenómeno socioeconómico no han disminuido las tasas de crecimiento o alterado la estabilidad política (Pesqué-Cela, 2018).

Así, los países que presentan una relación positiva entre IPC e IDH se ajustan al enfoque de los "sanders" que consideran a la corrupción como un obstáculo para el desarrollo; mientras que los países con una relación negativa entre IPC e IDH (incluido China), se alinean a los "greasers", que aceptan a la corrupción como incentivo para el desarrollo (Aidt, 2009).

Los resultados sugieren que hay elementos internos de cada país que pueden generar efectos diversos en el desarrollo económico. Lo que es similar a la idea de Becherair y Tahtane (2017), quienes establecen que el efecto de la corrupción sobre el IDH es a través de canales políticos. En concreto, los hallazgos están alineados al argumento de Kutan, Douglas y Judge (2007), quienes encuentran diferencias significativas en los efectos de la corrupción entre regiones de Asia y América Latina, concluyendo que la corrupción debe tratarse como un fenómeno de diferente importancia para ambas regiones. Por ejemplo, en China el tamaño de economía y dinamismo podría compensar los efectos negativos de la corrupción, en términos de desarrollo.

\section{Reflexiones finales}

Los resultados confirman la hipótesis planteada, esto es, elevados niveles de corrupción limitan el desarrollo económico - aproximado por el enfoque de desarrollo humano- Como corolario, se puede decir que la magnitud y frecuencia de los actos de corrupción amenazan las mejoras en el bienestar humano.

Los hallazgos sugieren que los procesos de desarrollo humano en economías grandes dentro de la región, como México, Argentina y Colombia reaccionan negativamente a la corrupción. Una menor percepción de este fenómeno se traduce en beneficios sociales. Al respecto, el enfoque de Aghion 
et al. (2016) es que una menor corrupción impulsa el crecimiento (PIB per cápita), el gasto en salud y en educación, lo que a su vez favorece los estándares de vida, la esperanza de vida y la acumulación de capital humano, y, en última instancia, el desarrollo humano. En este contexto, consideran que contener este fenómeno tiene el mayor impacto potencial para incrementar el bienestar social.

No obstante, para algunos países la relación es positiva. Particularmente para China y Brasil, economías grandes, global y regionalmente, la corrupción representa una herramienta que incentiva el desarrollo, lo que puede asociarse al tamaño del gobierno (Dzhumashev, 2014). Alternativamente, en estos países pueden coexistir corrupción y desarrollo, perpetuando el círculo perverso en términos de bienestar, lo que es similar a la idea de Tran (2008) sobre la existencia de equilibrios perversos en países pobres en el contexto de la corrupción.

La evidencia sugiere que los efectos de la corrupción son heterogéneos entre los países. Estas diferencias son atenuadas por otras variables que en conjunto afectan la sensibilidad del desarrollo a la corrupción. La magnitud de este efecto difiere entre países y regiones. Así, tamaño y dinamismo de la economía, una eficiente regulación económica, un gobierno con gasto público menor y un estado de derecho fuerte, que aumenten las oportunidades para las personas y disminuyan las opciones de distorsión del mercado y de corrupción, o que se castiguen actos relacionados, pueden disminuir los efectos negativos de ésta en el desarrollo.

Por ende, como la corrupción tiene impactos más que proporcionales en los países pobres y es corrosiva en la estructura misma de la sociedad, se reconoce como uno de los mayores desafíos en las economías consideradas, aun posiblemente representando beneficios para el desarrollo como en los casos de China y Brasil. Como es aún más complejo implementar políticas redistributivas sólidas para los países con altos niveles de corrupción, el impacto se refleja, además, en una reducción del valor presente de la inversión social. Así, la corrupción significa una barrera al desarrollo económico sostenible.

Bajo un ambiente de alta corrupción no es suficiente el combate puntual de la misma, ya que se requieren décadas de democracia para sacudirse las prácticas corruptas; las reglas de comportamiento social frente a este tema están mucho más arraigadas en la percepción de la corrupción que cualquier política estatal. En este sentido, como señalan Grossi y Pianezzi (2018), se 
requiere establecer un control democrático y construir una ética pública para el bien común.

El combate de la corrupción debe ser sostenible en el tiempo, ya que existe evidencia de que una caída en la corrupción sólo favorece el crecimiento económico si ha habido una disminución persistente de la corrupción (Swaleheen, 2012). Por tanto, sostener el desarrollo económico requiere de la reducción sistemática de la corrupción. Asimismo, esta lucha debe acompañarse de una reducción en la impunidad, ya que las políticas que imponen un fuerte costo moral a los evasores de impuestos y a los políticos corruptos pueden conducir a un único equilibrio óptimo (Litina y Palivos, 2016).

\section{Referencias}

Afonso, A., \& Tovar, J. (2011). Economic performance and government size (European Central Bank Working Paper Series 1399). Recuperado de https:// www.ecb.europa.eu/pub/pdf/scpwps/ecbwp1399.pdf

Aghion, P., Akcigit, U., Cagé, J., \& Kerr, W. (2016). Taxation, Corruption and Growth. European Economic Review, 86, 24-51. Recuperado de https://dash. harvard.edu/bitstream/handle/1/27420984/aghion,akcigit,cage,kerr_ taxation, corruption.pdf? sequence $=1$

Ahlin, C., \& Pang, J. (2008). Are financial development and corruption control substitutes in promoting growth? Journal of Development Economics, 86(2), 414.433. Recuperado de https://econpapers.repec.org/article/ eeedeveco/v_3a86_3ay_3a2008_3ai_3a2_3ap_3a414-433.htm

Ahmad, E., Aman, M., \& Arfeen, M. (2012). Does corruption affect economic growth? Latin American Journal of Economics, 49(2), 277-305. Recuperado de www.jstor.org/stable/41959246

Aidt, T. (2009). Corruption, institutions, and economic development. Oxford Review of Economic Policy, 25(2), 271-291. doi: https://doi.org/10.1093/ oxrep/grp012

Aidt, T., Dutta, J., \& Sena, V. (2008). Governance regimes, corruption and growth: Theory and evidence. Journal of Comparative Economics, 36(2), 195-220. doi: https://doi.org/10.1016/j.jce.2007.11.004

Akcay, S. (2006). Corruption and human development. Cato Journal, 26(1), 29-48. 
Akhter, S. H. (2004). Is globalization what it's cracked up to be? Economic freedom, corruption, and human development. Journal of World Business, 39(3), 283-295.

Alm, J., Martinez-Vazquez, J., \& McClellan C. (2016). Corruption and firm tax evasion. Journal of Economic Behavior \& Organization, 124, 46-163.

Alonso-Borrego, C., \& Arellano, M. (1996). Symmetrically Normalized Instrumental Variable Estimation Using Panel Data (CEMFI Working Paper 9612).

Alves, A., Costa, L., \& da Dias, L. (2017, April). Analysis of Correlation among HDI (Human Development Index), Violence and Corruption Perceptions Index. Presented at the Conference: 5th CR3 Making Corporate Responsibility Useful, Helsinki, Finland.

Arellano, M., \& Bond, S. (1991). Some Tests of Specification for Panel Data: Monte Carlo Evidence and an Application to Employment Equations. The Review of Economic Studies, 58(2), 277-297. doi: https://doi. org $/ 10.2307 / 2297968$

Arellano, M., \& Bover, O. (1995). Another look at the instrumental variable estimation of error-components models. Journal of Econometrics, 68(1), 29-51. doi: https://doi.org/10.1016/0304-4076(94)01642-D

Bahmani-Oskooee, M., \& Nasir, A. (2002). Corruption, Law and Order, Bureaucracy, and Real Exchange Rate. Economic Development and Cultural Change, 50(4), 1021-1028.

Balboa, J., \& Medalla, E. (2006, May). Anti-Corruption and Governance: The Philippine Experience. Presented at the APEC Study Center Consortium Conference, Ho Chi Mihn City, Viet Nam.

Beck, N., \& Katz, J. (1995). What to do (and not to do) with Time-Series Cross-Section Data. American Political Science Review, 89(3), 634-647. doi: https://doi.org/10.2307/2082979

Becherair, A., \& Tahtane, M. (2017). The causality between corruption and human development in MENA countries: A panel data analysis. EASTWEST Journal of ECONOMICS and BUSINESS, 20(2), 63-84.

Bechtel, G. (2018). The Human Development Index as Isoelastic GDP: Evidence from China and Pakistan. Economies, 6(2), 1-9. doi: https://doi. org/10.3390/economies6020032

Beltrán, A. (2016). Does corruption increase or decrease employment in firms? Applied Economic Letters, 23(5), 362-364. doi: https://doi.org/10.1080/1 3504851.2015.1076137 
Blackburn, K., \& Forgues-Puccio, G. (2010). Financial liberalization, bureaucratic corruption and economic development. Journal of International Money and Finance, 29(7), 1321-1339. doi: https://doi.org/10.1016/j. jimonfin.2010.05.002

Blackburn, K., \& Powell, J. (2011). Corruption, inflation and growth. Economic Letters, 113(3), 225-227. doi: https://doi.org/10.1016/j.econlet.2011.06.015

Blackwell, J. (2005). Estimation and testing of fixed-effect panel-data systems. The Stata Journal, 5(2), 202-207.

Blundell, R., \& Bond, S. (1998). Initial conditions and moment restrictions in dynamic panel data models. Journal of Econometrics, 87(1), 115-143. doi: https://doi.org/10.1016/S0304-4076(98)00009-8

Blundell, R., Bond, S., \& Windmeijer, F. (2000). "Estimation in Dynamic Panel Data Models: Improving on the Performance of the Standard GMM Estimator". En B. Baltagi, T. Fomby, \& C. Hill (Eds.), Nonstationary Panels, Panel Cointegration, and Dynamic Panels (Advances in Econometrics vol 15, pp.53-91). Nueva York: JAI Press. doi: https://doi.org/10.1016/ S0731-9053(00)15003-0

Boettke, P., \& Subrick, R. (2003). Rule of Law, Development, and Human Capabilities. Supreme Court Economic Review, 10, 109-126.

Bose, N., Capasso, S., \& Murshid, A. (2008). Threshold effects of corruption: theory and evidence. World Development, 36(7), 1173-1191. doi: https:// doi.org/10.1016/j.worlddev.2007.06.022

Carballo, A. (2010). Poverty and Corruption in Latin America: Challenges for a Sustainable Development Strategy. Opera, 10, 41-65.

Casas-Zamora, K., \& Carter, M. (2017). Beyond the scandals. The Changing Context of Corruption in Latin America. Washington, DC: Inter-American Dialogue. Recuperado de: https://www.thedialogue.org/analysis/beyondthe-scandals-the-changing-context-of-corruption-in-latin-america/

Chao, R. (29 de enero de 2013). Why Do Chinese Billionaires Keep Ending Up in Prison? The Atlantic. Recuperado de: https://www.theatlantic. com/international/archive/2013/01/why-do-chinese-billionaires-keepending-up-in-prison/272633/

Charoensukmongkol, P., \& Sexton, S. (2011). The Effect of Corruption on Exports and Imports in Latin America and the Caribbean. Latin American Business Review, 12(2), 83-98. doi: https://doi.org/10.1080/10978526. 2011.592800 
Cieslik, A., \& Goczek, L. (2018). Control of corruption, international investment, and economic growth. Evidence from panel data, World Development, 103,323-335. doi: https://doi.org/10.1016/j.worlddev.2017.10.028

Colonnelli, E., \& Prem, M. (2017). Corruption and firms: evidence from randomized audits in Brazil (SIEPR Working Paper 18-011). Recuperado de https://siepr.stanford.edu/sites/default/files/publications/18-011.pdf

Cooray, A., \& Dzhumashev, R. (2018). The effect of corruption on labour market outcomes. Economic Modelling, 74(C), 207-218. doi: https://doi. org/10.1016/j.econmod.2018.05.015

Cooray, A., Dzhumashev, R., \& Schneider, F. (2017). How Does Corruption Affect Public Debt? An Empirical Analysis. World Development, 90,115127. doi: https://doi.org/10.1016/j.worlddev.2016.08.020

Davies, A. (2009). Human development and the optimal size of government. The Journal of Socio-Economics, 38(2), 326-330. doi: https://doi. org/10.1016/j.socec.2008.07.015

Davies, A., \& Quinlivan, G. (2006). A panel data analysis of the impact of trade on human development. The Journal of Socio-Economics, 35(5), 868-876. doi: https://doi.org/10.1016/j.socec.2005.11.048

Delgado, M., McCloud, N., \& Kumbhakar, S. (2014). A Generalized Empirical Model of Corruption, Foreign Direct Investment and Growth. Journal of Macroeconomics, 42, 298-316.

Deysine, A. (1980). Political Corruption: A review of the literature. European Journal of Political Research, 8(4), 447-462. doi: https://doi. org/10.1111/j.1475-6765.1980.tb00583.x

du Plessis, P. (2014). Corruption in Education. Stealing the Future. Mediterranean Journal of Social Sciences, 5(23), 1308-1316. doi: https://doi. org/10.5901/mjss.2014.v5n23p1308

Dzhumashev, R. (2014). Corruption and growth: The role of governance, public spending, and economic development. Economic Modelling, 37, 202-215. doi: https://doi.org/10.1016/j.econmod.2013.11.007

Everhart, S., Martinez-Vazquez, J., \& McNab, R. (2003). Corruption, investment, and growth in developing countries. $96^{\text {th }}$ Proceedings. Annual Conference on Taxation and Minutes of the Annual Meeting of the National Tax Association (pp.84-90). Washington DC, United States of America.

Frisch, D. (1996). The effects of corruption on development. The Courier, 158(4), 68-70.

Greene, W. (2012). Econometric Analysis. Nueva York: Prentice Hall. 
Grossi, G., \& Pianezzi, D. (2018). The new public corruption: Old questions for new challenges. Accounting Forum, 42(1), 86-101. doi: https://doi. org/10.1016/j.accfor.2016.05.002

Haggard, S., MacIntyre, A., \& Tiede, L. (2008). The Rule of Law and Economic Development. Annual Review of Political Science, 11, 205-234. doi: https:// doi.org/10.1146/annurev.polisci.10.081205.100244

Haidar, J. (2012). The impact of business regulatory reforms on economic growth. Journal of the Japanese and International Economies, 26(3), 285307. doi: https://doi.org/10.1016/j.jjie.2012.05.004

Henderson, D. (19 de abril de 1999). Power corrupts. Editorial comment. The Wall Street Journal.

Hoechle, D. (2007). Robust standard errors for panel regressions with crosssectional dependence. The Stata Journal, 7(3), 281-312.

Hoinaru, R., Buda, D., Borlea, S., Văidean, V., \& Achim, M. (2020). The Impact of Corruption and Shadow Economy on the Economic and Sustainable Development. Do They "Sand the Wheels" or "Grease the Wheels"? Sustainability, 12(2), 481. doi: https://doi.org/10.3390/su12020481

Hopkin, J., \& Rodríguez-Pose, A. (2007). “Grabbing Hand" or "Helping Hand”? Corruption and the Economic Role of the State. Governance, 20(2), 187208. doi: https://doi.org/10.1111/j.1468-0491.2007.00353.x

Huang, C. (2016). Is corruption bad for economic growth? Evidence from Asia-Pacific countries. The North American Journal of Economics and Finance, 35, 247-256. doi: https://doi.org/10.1016/j.najef.2015.10.013

Jain, P., Kuvvet, E., \& Pagano, M. (2017). Corruption's impact on foreign portfolio investment. International Business Review, 26(1), 23-35. doi: https://doi.org/10.1016/j.ibusrev.2016.05.004

Jalilian, H., Kirkpatrick, C., \& Parker, D. (2007). The Impact of Regulation on Economic Growth in Developing Countries: A Cross-Country Analysis. World Development, 35(1), 87-103. doi: https://doi.org/10.1016/j. worlddev.2006.09.005

Jiang, T., \& Nie, H. (2014). The stained China miracle: Corruption, regulation, and firm performance. Economic Letters, 123(3), 366-369. doi: https:// doi.org/10.1016/j.econlet.2014.03.026

Justesen, M., \& Bjornskov, C. (2014). Exploiting the Poor: Bureaucratic Corruption and Poverty in Africa. World Development, 58, 106-115. doi: https://doi.org/10.1016/j.worlddev.2014.01.002 
Kar, S., \& Saha, S. (2012). Corruption, Shadow Economy and Income Inequality: Evidence from Asia (IZA Discussion Papers No. 7106).

Kutan, A., Douglas, T., \& Judge, W. (2007). Does Corruption Hurt Economic Development? Evidence from Middle Eastern, North African and Latin American Countries. Edwardsville: Southern Illinois University.

Larsson, T. (2006). Reform, corruption and growth: Why corruption is more devastating in Russia than in China. Communist and Post-Communist Studies, 39(2), 265-281. doi: https://doi.org/10.1016/j.postcomstud.2006.03.005

Lash, N. (2004). Corruption and Economic Development. The Journal of Economic Asymmetries, 1(1), 85-109. doi: https://doi.org/10.1016/j. jeca.2004.01.007

Lederman, D., Loayza, N., \& Soares, R. (2005). Accountability and Corruption: Political Institutions Matter. Economics \& Politics, 17(1), 1-35.

Lewis, M. (2006). Governance and Corruption in Public Health Care Systems (Center for Global Development Working Paper 78).

Litina, A., \& Palivos, T. (2016). "Corruption, Tax Evasion and Social Values". Journal of Economic Behavior \& Organization, 124(C), 164-177. doi: https:// doi.org/10.1016/j.jebo.2015.09.017

Lopez, J. (2019). Corruption and its Effect on Economic Development in Chile, Mexico, and Brazil. Governance: The Political Science Journal at UNLV, 6. Recuperado de: https://digitalscholarship.unlv.edu/governance-unlv/ vol6/iss1/4/

Lucic, D., Radisic, M., \& Dobromirov, D. (2016). Causality between corruption and the level of GDP. Economic Research, 29(1), 360-379. doi: https://doi. org/10.1080/1331677X.2016.1169701

Martins, S., \& Vega, J. (2013). Government size, composition of public expenditure, and economic development (NIPE Working Papers 17/2013).

Méndez, F., \& Sepúlveda, F. (2006). Corruption, growth and political regimes: Cross country evidence. European Journal of Political Economy, 22(1), 8298. doi: https://doi.org/10.1016/j.ejpoleco.2005.04.005

Mo, P. (2001). Corruption and Economic Growth. Journal of Comparative Economics, 29(1), 66-79. doi: https://doi.org/10.1006/jcec.2000.1703

Organización para la Cooperación y el Desarrollo Económicos (OCDE). (2013). Corruption. Organización para la Cooperación y Desarrollo Económicos: París. Recuperado de http://www.oecd.org/corruption/antibribery/39532693.pdf 
Özsahin, S., \& Üçler, G. (2017). The Consequences of Corruption on Inflation in Developing Countries: Evidence from Panel Cointegration and Causality Tests. Economies, 5(49), 1-15. doi: https://doi.org/10.3390/ economies5040049

Pesqué-Cela, V. (2018). Corruption in China. En B. Warf, (Ed.), Handbook on the geographies of corruption (313-330). Chletenham: Edward Elgar Publishing. Petrou, A., \& Thanos, I. (2014). The "grabbing hand" or the "helping hand" view of corruption: Evidence from bank foreign market entries. Journal of World Business, 49(3), 444-454. doi: https://doi.org/10.1016/j.jwb.2013.10.004

Pieroni, L., \& d'Agostino, G. (2013). Corruption and the effects of economic freedom. European Journal of Political Economy, 29, 54-72. doi: https:// doi.org/10.1016/j.ejpoleco.2012.08.002

Pignataro, A. (2018). Análisis de datos de panel en ciencia política: ventajas y aplicaciones en estudios electorales. Revista Española de Ciencia Política, 46,259-283. doi: https://doi.org/10.21308/recp.46.11

Pilapitiya, T. (2004). The impact of corruption on the human rights-based approach to development. Oslo: United Nations Development Program / Oslo Governance Centre.

Programa de las Naciones Unidas para el Desarrollo (PNUD). (2016). Informe sobre Desarrollo Humano 2016. Desarrollo Humano para todas las personas. Nueva York: Programa de las Naciones Unidas para el Desarrollo. Recuperado de: http://hdr.undp.org/sites/default/files/HDR2016_SP_Overview_Web_0.pdf

Programa de las Naciones Unidas para el Desarrollo (PNUD). (2019). Informe sobre Desarrollo Humano 2019. Más allá del ingreso, más allá de los promedios, más allá del presente: Desigualdades del desarrollo humano en el siglo XXI. Nueva York: Programa de las Naciones Unidas para el Desarrollo. Recuperado de: http://hdr.undp.org/sites/default/files/hdr_2019_overview_-_spanish.pdf

Programa de las Naciones Unidas para el Desarrollo (PNUD). (2020). Reportes del Desarrollo Humano. Programa de las Naciones Unidas para el Desarrollo. Recuperado de http://hdr.undp.org/en/data

Policardo, L., \& Sánchez, E. (2018). Corruption causes inequality, or is it the other way around? An empirical investigation for a panel of countries. Economic Analysis and Policy, 59(C), 92-102. doi: https://doi.org/10.1016/j. eap.2018.05.001 
Popova, Y., \& Podolyakina, N. (2014). Pervasive impact of corruption on social system and economic growth. Procedia. Social and Behavioral Sciences, 110, 727-737. doi: https://doi.org/10.1016/j.sbspro.2013.12.917

Quazi, R., Vemuri, V., \& Soliman, M. (2014). Impact of Corruption on Foreign Direct Investment in Africa. International Business Research, 7(4), 1-10. doi: https://doi.org/10.5539/ibr.v7n4p1

Ranis, G. (2004). Human development and economic growth. (Economic Growth Center, Yale University, Center Discussion Paper 887).

Rock, M. \& Bonnett, H. (2004). The Comparative Politics of Corruption: Accounting for the East Asian Paradox in Empirical Studies of Corruption, Growth and Investment. World Development, 32(6), 999-1017. doi: https://doi.org/10.1016/j.worlddev.2003.12.002

Rose-Ackerman, S. (2008). Corruption and Government. International Peacekeeping Journal, 15(3), 328-343. doi: https://doi. org/10.1080/13533310802058802

Rosen, N. (23 de noviembre de 2012). From rich list to death list, China's road to wealth. BBCNews. Recuperado de: https://www.bbc.com/news/ business-20454207

Saha, S., \& Gounder, R. (2013). Corruption and economic development nexus: Variations across income level in a non-linear framework. Economic Modelling, 31, 70-79. doi: https://doi.org/10.1016/j.econmod.2012.11.012

Swaleheen, M. (2012). Curbing corruption for higher growth: The importance of persistence. Economic Letters, 116(2), 255-257. doi: https://doi. org/10.1016/j.econlet.2012.03.011

Svensson, J. (2005). Eight Questions about Corruption. Journal of Economic Perspectives, 19(3), 19-42. doi: https://doi.org/10.1257/089533005774357860

Tanzi, V. (1998). Corruption Around the World: Causes, Consequences, Scope and Cures (International Monetary Fund Working Paper 98/63). Recuperado de: https://www.imf.org/external/pubs/ft/wp/wp9863.pdf

Talvitie, A. (2017). Observed Differences in Corruption between Asia and Africa: The Industrial Organization of Corruption and Its Cure. Transportation Research Procedia, 25, 4472-4490. doi: https://doi.org/10.1016/j. trpro.2017.05.357

Teker, S., \& Güner, A. (2016). Whether Development Indices Affect Economic Growth: A Cross-Country Analysis. Procedia Economics and Finance, 38, 340-346. doi: https://doi.org/10.1016/S2212-5671(16)30206-4 
The Heritage Foundation. (2020). Index of Economic Freedom. Recuperado de https://www.heritage.org/

Tiberiu, C. Tamasila, M., \& Taucean, I. (2016). Entrepreneurship, Tax Evasion and Corruption in Europe. Social and Behavioral Sciences, 221, 246-253. doi: https://doi.org/10.1016/j.sbspro.2016.05.112

Tran, N. (2008). Corruption and human development (Development and Policies Research Center DEPOCEN Working Paper 7).

Trang, H., Papanastassiou, M., \& Nguyen, Q. (2017). Multinationals and the impact of corruption on financial derivatives use and firm value: Evidence from East Asia. Journal of Multinational Financial Management, 39, 39-59. doi: https://doi.org/10.1016/j.mulfin.2017.02.001

Transparency International. (2020). Corruption Perceptions Index. Recuperado de https://www.transparency.org/es/cpi/

United Nations. (2004). United Nations Convention Against Corruption. Austria: United Nations. Recuperado de: https://www.unodc.org/documents/ brussels/UN_Convention_Against_Corruption.pdf

Wang, Y., \& You, J. (2012). Corruption and firm growth: Evidence from China. China Economic Review, 23(3), 415-433. doi: https://doi.org/10.1016/j. chieco.2012.03.003

Wooldridge, J. (2002). Econometric Analysis of Cross Section and Panel Data. Cambridge: MIT Press.

Wong, M. (2016). Public spending, corruption, and income inequality: A comparative analysis of Asia and Latin America. International Political Science Review, 38(3), 298-315. doi: https://doi.org/10.1177/0192512116642617

World Bank. (1997). Helping Countries Combat Corruption: The Role of the World Bank. Washington: World Bank.

World Bank. (2018). World Development Report 2018 (WDR 2018)-LEARNING to Realize Educations' Promise. Washington: World Bank. doi: https://doi. org/10.1596/978-1-4648-1096-1

World Data Bank. (2020). Databases. Recuperado de https://databank.worldbank.org/databases

Wu, S., Li, B., Nie, Q., \& Chen, C. (2017). Government expenditure, corruption and total factor productivity. Journal of Cleaner Production, 168, 279-289. doi: https://doi.org/10.1016/j.jclepro.2017.09.043

Yao, S. (2002). Privilege and Corruption: The Problems of China's Socialist Market Economy. The American Journal of Economics and Sociology, 61(1), 279-299. Doi: https://doi.org/10.1111/1536-7150.00160 
Yilmaz, I. \& Tag, M. (2016). Well-Being of Society: The Role of Institutions vs. Government Expenditure. The European Proceedings of Social \& Behavioural Sciences, VII, 132-143. Recuperado de https://www.futureacademy.org. uk/files/images/upload/_WELLSO_2015.pdf

Yong, G. (2008). Corruption in Transitional China: An Empirical Analysis. The China Quarterly, 194, 349-364. 